\title{
Weakly Pulse-Coupled Oscillators, FM Interactions, Synchronization, and Oscillatory Associative Memory
}

\author{
Eugene M. Izhikevich
}

\begin{abstract}
We study pulse-coupled neural networks that satisfy only two assumptions: each isolated neuron fires periodically, and the neurons are weakly connected. Each such network can be transformed by a piece-wise continuous change of variables into a phase model, whose synchronization behavior and oscillatory associative properties are easier to analyze and understand. Using the phase model, we can predict whether a given pulse-coupled network has oscillatory associative memory, or what minimal adjustments should be made so that it can acquire memory. In the search for such minimal adjustments we obtain a large class of simple pulse-coupled neural networks that can memorize and reproduce synchronized temporal patterns the same way a Hopfield network does with static patterns. The learning occurs via modification of synaptic weights and/or synaptic transmission delays.
\end{abstract}

Index Terms - Canonical models, Class 1 neural excitability, integrate-and-fire neurons, multiplexing, syn-fire chain, transmission delay.

\section{INTRODUCTION}

$\mathbf{I}$ $\mathrm{N}$ THIS turorial paper we exploit the relationship between pulse-coupled neural networks and phase models [3], [20], [34]. Since synchronization behavior and some oscillatory associative properties of phase models are understood (see [14] for a review), the relationship provides a powerful tool for analysis of pulse-coupled networks.

Since we do not assume that the reader is familiar with phase models, we devote a large portion of the paper (Sections IV and VI) to an elementary introduction into some essential aspects of the phase model theory.

\section{A. Pulse-Coupled Neural Networks}

Some treat pulse-coupled networks as the third generation of neural-network models that take into account the spiking nature of neurons, and hence, are much closer to biology than classical artificial neural network models (see, e.g., [22] and [23] for a review). Many other scientists, especially those who study Hodgkin-Huxley neurons, treat pulse-coupled neural networks as toy models; that is, even though the networks are based on abstractions of important properties of biological neurons, they are still far away from the reality (despite the fact that we have no idea what the reality is). As a conse-

Manuscript received August 8, 1997; revised November 1, 1998.

The author is with the Center for Systems Science and Engineering, Arizona State University, Tempe, AZ 85287-7606 USA.

Publisher Item Identifier S 1045-9227(99)03187-2. quence, all results obtained by studying pulse-coupled neural networks might be irrelevant to the brain. However, it has been proven recently [14], [19] that many biophysically detailed and biologically plausible Hodgkin-Huxley-type neural networks can be transformed into pulse-coupled form by a piece-wise continuous change of variables. Thus, the difference between pulse-coupled neural networks and the Hodgkin-Huxley-type models is just a matter of coordinate change. Therefore, it is imperative that we understand the information processing that takes place in pulse-coupled neural networks.

\section{B. Review of Methods}

There are many approaches toward understanding behavior of pulse-coupled neural networks:

- Spike Density Distribution: Methods of statistical physics, such as Fokker-Planck equation and mean-field approximations, have proven to be useful in analysis of spike density distributions of fully connected pulse-coupled oscillatory networks when the size of the network $n \rightarrow \infty$ and the strength of connections $\varepsilon=1 / n \rightarrow 0$; see [1], [20], and [33].

- Spike Response Models: Many weakly pulse-coupled networks can be written in the "spike response" form, which is a system of nonlinear integral equations having "synaptic kernels" (see review by W. Gerstner [9]).

- Firing Maps: Analysis of strongly pulse-coupled networks can be reduced to an analysis of a firing mapping. The theory is based on the Poincaré return maps, and was first introduced by Mirollo and Strogatz [28]; see also [8], [25], and [29].

- Phase Models: Weakly pulse-coupled networks of oscillatory neurons can be transformed into simpler phase models [3], [20], [34]. This is the approach we use in the present paper.

Of course, a straightforward analysis [3], [12] and computer simulations can also provide an invaluable information about behavior of pulse-coupled networks.

\section{Weak Connections}

Most of the methods mentioned above work only when connections between neurons are weak. Remarkably, this assumption is biologically plausible, since it follows from in vitro observation that amplitudes of postsynaptic potentials are less than $1 \mathrm{mV}$, which is extremely small in comparison with 
the amplitude of an action potential (around $100 \mathrm{mV}$ ) and the amplitude of the mean EPSP necessary to discharge a quiescent cell (around $20 \mathrm{mV}$ ); see [14, Sec. 1.3].

For example, PSP's in hippocampal granule cells are as small as $0.1 \pm 0.03 \mathrm{mV}$ [26]. Firing of a hippocampal CA3 pyramidal cell elicits EPSP in other CA3 pyramidal cells ranging from 0.6 to $1.3 \mathrm{mV}$ [27]. Firing of the same CA3 cell can evoke EPSP in CA1 pyramidal cells (via Schaffer collateral) of amplitude $0.13 \mathrm{mV}$ [31]. The majority of PSP's in pyramidal neurons of the rat visual cortex are less than $0.5 \mathrm{mV}$ in amplitude, with the range $0.05-2.08 \mathrm{mV}$ [24]. As Mason and coauthors [24] point out in their discussion section, there is an underestimate of the true range because PSP's smaller than $0.03 \mathrm{mV}$ would have gone undetected.

\section{Phase Model}

If the pulse-coupled neurons are weakly connected and each neuron exhibits autonomous oscillatory behavior, then the entire network can be transformed into a simpler phase model by a piece-wise continuous change of variables. A precise statement is given by Theorem 1 whose proof can be found in the Appendix. A generalization of the theorem that takes into account nontrivial temporal dynamics of synaptic transmission is straightforward [34] and can also be found in Appendix II.

Each (phase) variable in the phase model has the meaning of timing of firing of the corresponding neuron, and the phase model captures how timing of one neuron affects that of the other one on a long time scale. The phase model is not pulse-coupled, which simplifies its analysis significantly. Still, studying the phase model provides an invaluable information about pulse-coupled networks because the difference between the former and the latter is just a matter of coordinate change.

\section{E. FM Interactions}

If the weakly pulse-coupled network consists of neurons having nonresonant frequencies, then the corresponding phase model is uncoupled. Thus, the long-term interactions between nonresonant neurons can be "removed" by a piece-wise continuous change of variables. This mathematical result can be explained in ordinary language as follows: Since the neurons are weakly connected, any change in timing of firing of one cell induces small changes in timing of another cell. Such small changes can accumulate and become significant on a time scale of many periods, or they can cancel each other and remain small. When the neurons have nonresonant frequencies, the changes cancel each other. In contrast, when the neurons have resonant or nearly equal frequencies, the small changes accumulate and become significant.

We see that whether or not two neurons interact depends not only on the existence of synaptic connections between them, but also on the relation between their frequencies. The interaction is most effective when the frequencies are nearly equal. We refer to such interactions as being frequency modulated (FM) and discuss them in detail in Section III, which is aimed to the readers having no mathematical background. There we discuss how FM interactions can provide a powerful mechanism for multiplexing of neural signals.

\section{F. Synchronization}

Studying synchronization and other locking behavior in pulse-coupled networks can be a daunting task. In Section IV we show that it is a relatively simple task when we consider phase models. Our major tool is the Ermentrout theorem [6], which provides sufficient conditions for stability of synchronized solutions. In Section VII we use the classical integrateand-fire model to illustrate how the theorem works. Our analytical results are corroborated by computer simulations and findings of other scientists who used alternative methods discussed in Section I-B above. In particular, we confirm a well-known fact that neither excitation, inhibition, nor synaptic transmission delay alone contributes to synchronization, but their interplay does.

\section{G. Oscillatory Associative Memory}

A sufficient condition for the existence of oscillatory associative memory in phase models is well known. It follows from the Theorem 9.15 by Hoppensteadt and Izhikevich [14], which we present in Section VI. There we extend the theorem for pulse-coupled neurons. Thus, we obtain a simple criterion to determine whether a given pulse-coupled network has the associative memory. It turns out that the leaky integrate-andfire model does not satisfy the criterion. In attempt to make a minimal adjustment, we discover a large class of simple pulse-coupled networks that are guaranteed to have oscillatory associative memory: They can memorize and reproduce nontrivial temporal patterns in the same way the standard Hopfield network does with static patterns.

\section{H. Learning Through Modification of Transmission Delays}

Connections between pulse-coupled neurons are determined by two sets of parameters: the strengths and the delays. It is commonly assumed that learning consists in modifying the strength of connections, while the delays are either kept constant or neglected. In Section VII we present a (complexconjugate Hebbian) learning rule that changes the delays to memorize temporal patterns. The learning rule was derived originally for weakly connected oscillators near multiple Andronov-Hopf bifurcation [16], and it seems to work for pulse-coupled oscillatory networks too.

\section{The Phase Model}

\section{A. General Pulse-Coupled Neural Networks}

Many pulse-coupled networks can be written in the following form:

$$
\dot{x}_{i}=f_{i}\left(x_{i}\right)+\varepsilon \sum_{j=1}^{n} g_{i j}\left(x_{i}\right) \delta\left(t-t_{j}^{*}-\eta_{i j}\right) .
$$

Here $x_{i} \in[0,1]$ denotes the rescaled membrane potential of the $i$ th neuron. The function $f_{i}$ describes its dynamics. When $x_{i}$ reaches 1 , the $i$ th neuron is said to fire a spike, and $x_{i}$ is reset to the new value $x_{i}=0$. This moment is marked as $t_{i}^{*}$. The dimensionless parameter $\varepsilon$ denotes the strength of connections in the network. The function $g_{i j}$ describes the effect of firing 
of the $j$ th neuron onto the $i$ th one: If $x_{j}$ fires, variable $x_{i}$ is incremented by $\varepsilon g_{i j}\left(x_{i}\right)$ after some time delay $\eta_{i j} \geq 0$. The increment is produced by the Dirac delta function $\delta$ satisfying $\delta(t)=0$ for all $t \neq 0, \delta(0)=\infty$, and $\int \delta=1$. Neither $f_{i}$ nor $g_{i j}$ are assumed to be continuous. A possible ambiguity of the pulse-coupled network of the form (1) when two or more neurons fire simultaneously is discussed in Appendix I-A.

A typical example of a pulse-coupled neural network is the leaky integrate-and-fire model, which can be written in the form (1) for

$$
f_{i}\left(x_{i}\right)=a_{i}-b_{i} x_{i} \quad \text { and } \quad g_{i j}\left(x_{i}\right)=s_{i j}
$$

where $s_{i j} \in \mathbb{R}$ are some (synaptic) coefficients. A nontrivial $g_{i j}$ arises when the absolute refractory period is taken into account; that is, the period immediately after the firing during which the neuron is not sensitive to any input. Let $x_{\mathrm{ref}}<1$ be the length of the refractory period, then

$$
g_{i j}\left(x_{i}\right)= \begin{cases}0, & \text { if } x_{i} \in\left[0, x_{\mathrm{ref}}\right), \\ s_{i j}, & \text { if } x_{i} \in\left[x_{\mathrm{ref}}, 1\right) .\end{cases}
$$

\section{B. Weakly Connected Neural Networks}

In this paper we assume that $\varepsilon \ll 1$, which implies that connections between neurons are weak. This assumption follows from in vitro observation that amplitudes of postsynaptic potentials (PSP's) are around $0.1 \mathrm{mV}$, which is small in comparison with the amplitude of an action potential (around $100 \mathrm{mV}$ ) and the amplitude of the mean EPSP necessary to discharge a quiescent cell (around $20 \mathrm{mV}$ ); see detailed discussion by Hoppensteadt and Izhikevich [14, Sec. 1.3] who obtained an estimate $0.004<\varepsilon<0.008$ for a model of hippocampal granule cells using in vitro data from hippocampus [26].

We remark that we do not need infinitesimal $\varepsilon$ for our manipulations below. For example, the phase model theorem " 1 " is expressed in the form: There is an $\varepsilon_{0}>0$ such that for all $\varepsilon \leq \varepsilon_{0} \cdots$. Most of the illustrations found in this paper are simulations of integrate-and-fire networks for $\varepsilon=1 / 20$.

\section{Periodic Spiking}

We are interested in the case when each neuron

$$
\dot{x}_{i}=f_{i}\left(x_{i}\right)
$$

in the pulse-coupled network (1) can fire repeatedly without any input from the other neurons. This happens when $f_{i}\left(x_{i}\right)>$ 0 for all $x_{i} \in[0,1]$. [Indeed, if $f_{i}\left(x_{0}\right)=0$ for some $x_{0} \in[0,1]$, then $x_{i}$ stops at $x_{i}=x_{0}$.] One can easily find the period

$$
T_{i}=\int_{0}^{1} \frac{d x}{f_{i}(x)}
$$

and the frequency

$$
\Omega_{i}=\frac{2 \pi}{T_{i}}
$$

of such periodic spiking.

\section{The Phase Model Theorem}

Let $X_{i}(t)$ be the solution of the $i$ th equation in the uncoupled system (3) starting from $X_{i}(0)=0$; that is,

$$
\dot{X}_{i}=f_{i}\left(X_{i}\right), \quad X_{i}(0)=0 .
$$

Obviously, $X_{i}(t)$ is $T_{i}$-periodic, and its firing occurs at the times $T_{i}, 2 T_{i}, 3 T_{i}, \cdots$. Weak input from the other neurons may delay or advance each firing thereby introducing an $\varepsilon$ phase deviation (phase shift). Such deviations may accumulate with each cycle and become significant on the time scale of order $1 / \varepsilon$. To take them into account, we introduce a slow phase deviation variable $\varphi_{i} \in \mathbb{S}^{1}$. Here $\mathbb{S}^{1}$ is the unit circle, which can be thought of as the interval $[0,2 \pi]$ having points zero and $2 \pi$ identified (glued).

Theorem 1-Phase Model For Weakly Pulse-Coupled Oscillators: Consider the pulse-coupled system of the form (1) and suppose that each $f_{i}>0$; that is, each neuron fires repeatedly. Suppose that each synaptic transmission delay $\eta_{i j}$ is a constant that is independent from the strength of connections $\varepsilon$. Then, there is an $\varepsilon_{0}>0$ such that for all $\varepsilon \leq \varepsilon_{0}$ there is a piece-wise continuous change of variables of the form

$$
x_{i}(t)=X_{i}\left(t+\Omega_{i}^{-1} \varphi_{i}(\varepsilon t)\right)+\mathcal{O}(\varepsilon)
$$

that transforms (1) into the phase model

$$
\varphi_{i}^{\prime}=\omega_{i}+\sum_{j=1}^{n} H_{i j}\left(\varphi_{i}, \varphi_{j}\right)+\mathcal{O}(\varepsilon)
$$

where $^{\prime}=d / d \tau$ and $\tau=\varepsilon t$ is the slow time. Each parameter

$$
\omega_{i}=\sum_{j=1}^{n} h_{i j}^{0}
$$

accounts for frequency changes that are due to network connections [constants $h_{i j}^{0}$ are defined below in (9)]. Each connection function $H_{i j}$ has zero mean value and can be written in one of the following forms:

- Equal Frequencies: If $\Omega_{i}=\Omega_{j}$, then

$$
H_{i j}\left(\varphi_{i}, \varphi_{j}\right)=\frac{1}{2 \pi} h_{i j}\left(\psi_{i j}+\varphi_{i}-\varphi_{j}\right)-h_{i j}^{0}
$$

where $\psi_{i j}=\Omega_{j} \eta_{i j}$ is the rescaled time delay

$$
h_{i j}(\theta)=\Omega_{i} \Omega_{j} \frac{g_{i j}\left(X_{i}\left(\Omega_{i}^{-1} \theta\right)\right)}{f_{i}\left(X_{i}\left(\Omega_{i}^{-1} \theta\right)\right)}
$$

is a function, and

$$
h_{i j}^{0}=\frac{1}{(2 \pi)^{2}} \int_{0}^{2 \pi} h_{i j}(\theta) d \theta
$$

is a constant.

- Resonant Frequencies: If $p \Omega_{i}=q \Omega_{j}$ for some relatively prime integers $p$ and $q$, then

$$
H_{i j}\left(\varphi_{i}, \varphi_{j}\right)=Q_{i j}\left(p \varphi_{i}-q \varphi_{j}\right)
$$

for some $2 \pi$-periodic function $Q_{i j}$ that vanishes when the order of resonance, $|p+q|$, increases. 


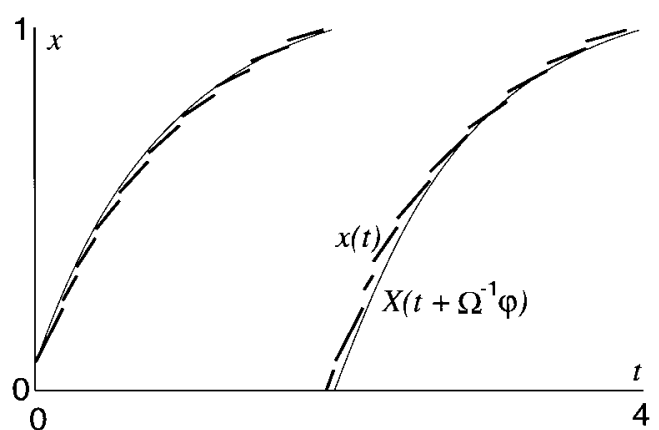

Fig. 1. An illustration of the relation (5) between the actual solution, $x(t)$, of the pulse-coupled system (1) and its reconstruction using the phase model (6). Simulations of the leaky integrate-and-fire neurons, $a_{i}=1, b_{i}=0.9, \varepsilon=0.02$; see Section V.

- Nonresonant Frequencies: If $p \Omega_{i} \neq q \Omega_{j}$ for all nonzero integers $p$ and $q$, then

$$
H_{i j}\left(\varphi_{i}, \varphi_{j}\right)=0
$$

for all $\varphi_{i}$ and $\varphi_{j}$.

The proof of the theorem involves averaging theory and is given in Appendix I. A generalization of the theorem for the case of nontrivial temporal synaptic transmission is discussed in Appendix II.

In this paper we consider weakly pulse-coupled oscillators having equal frequencies and possibly delayed interactions. In this case function $H_{i j}$ is given by (7). Obviously, it depends on the phase difference $\varphi_{i}-\varphi_{j}$, so that we can rewrite the phase model (6) in the following "classical" form

$$
\varphi_{i}^{\prime}=\omega_{i}+\sum_{j=1}^{n} H_{i j}\left(\varphi_{j}-\varphi_{i}\right)
$$

Since we neglect the small order term $\mathcal{O}(\varepsilon)$, the relation (5) illustrated in Fig. 1 is valid only on the fast time scale, $t$, of order $1 / \varepsilon$. The relation may hold on the infinite time scale if additional conditions are imposed, e.g., that (10) exhibits frequency locking.

\section{FM INTERACTIONS AND THE NATURE OF NEURAL CODE}

We see that the pulse-coupled neural network (1) can be transformed into the phase model (6) by the change of variables of the form (5). Synaptic connections from the $j$ th neuron to the $i$ th one affect the value of the parameter $\omega_{i}$ and the form of the function $H_{i j}$. The former determines the "averaged" effect of the connection that is due to increased excitation (or inhibition) converging to the $i$ th neuron. The latter determines how the phase (timing of firing) of one neuron affects that of the other one. We say that two neurons interact when one of them can distinguish timing of another one.

The form of $H_{i j}$ depends on the relation between the frequencies $\Omega_{i}$ and $\Omega_{j}$. A seemingly counterintuitive fact is that $H_{i j} \equiv 0$ when the frequencies are nonresonant. It implies that whether or not the neurons interact depends not only on the existence of synaptic connections between them, but also on the relation between their frequencies. A neuron can
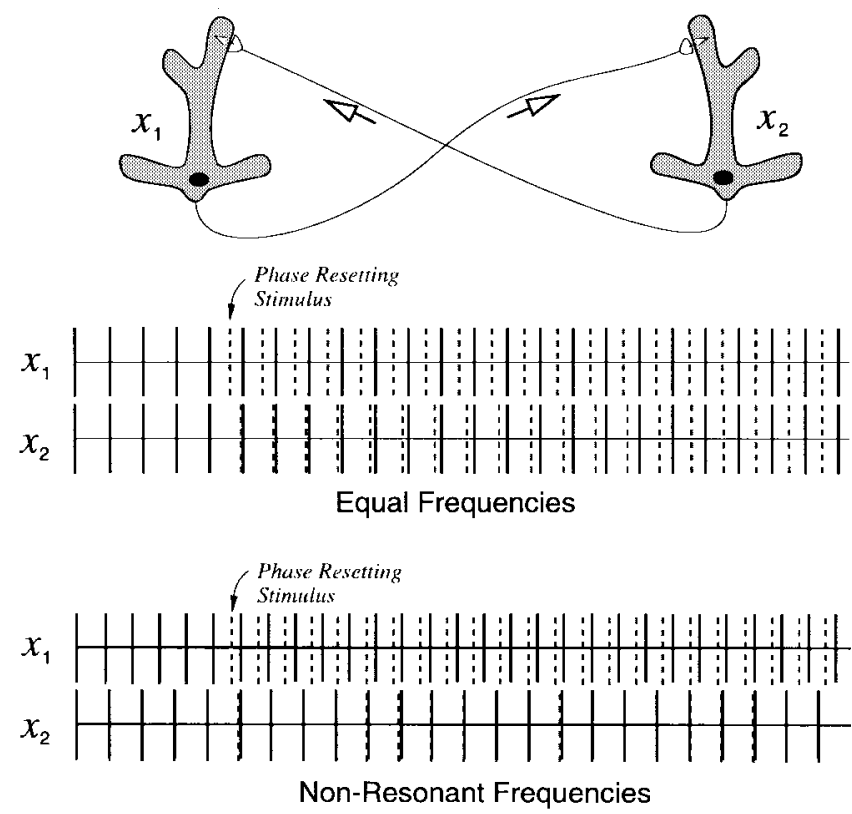

Fig. 2. An illustration of FM interactions between two neurons: Whether or not two interconnected neurons interact depends on the ratio of their frequencies. Continuous bars denote the spiking activity of two neurons. Dashed vertical bars denote firing when a brief perturbation is applied. Equal Frequencies: Any changes in phase (timing) of firing of one neuron affect the phase (timing) of the other one. Nonresonant Frequencies: A neuron is not sensitive to changes in phase of the other neuron even though the neurons are connected. (Simulations of two leaky integrate-and-fire neurons. Parameters: $\varepsilon=0.05, t \in[0,30], b_{i}=0.5, g_{i j}=1, a_{1}=1, a_{2}=1$, or $a_{2}=1.15$.)

dynamically turn on or off its communication with another neuron simply by changing the frequency of spiking, without changing the strength of synaptic connections $g_{i j}$.

We illustrate this issue in Fig. 2 using two coupled integrateand-fire neurons. When they have equal frequencies, they lock (see continuous vertical bars in the upper part of the figure). If a brief strong stimulus is applied to $x_{1}$ so that its phase is shifted (dashed bars), the other neuron tracks the change by acquiring a phase shift (compare continuous and dashed bars for $\left.x_{2}\right)$. Since the neurons are weakly connected $(\varepsilon=1 / 20)$, the interaction between them is not instantaneous; i.e., it takes a few spikes, but the neurons do communicate via their phases. In contrast, when they have nonresonant frequencies, one of the neurons is not sensitive to phase of the other one. Such neurons do not interact, at least on the time scale of $1 / \varepsilon$ periods.

In Fig. 3 we consider another, less trivial example. We model each column by three strongly connected leaky integrate-and-fire neurons so that $x_{i 3}$ fires whenever $x_{i 1}$ or $x_{i 2}$ do. Thus, the spike train of $x_{i 3}$ is a superposition of the spike trains of $x_{i 1}$ and $x_{i 2}$. The latter fire periodically with the frequencies $\omega_{i 1}$ and $\omega_{i 2}$, respectively, where $i=1$ or 2 is the column number. We also assume that $\omega_{11}=\omega_{21}$ and $\omega_{12}=\omega_{22}$. Notice that both neurons $x_{21}$ and $x_{22}$ in the second column receive an identical (quasiperiodic) spike train from the first column via $x_{13}(t)$. From Theorem 1 it follows that $x_{21}$ can distinguish the part of the signal coming from $x_{11}$ from the part coming from $x_{12}$, because $x_{21}$ and $x_{11}$ have the same frequencies. To verify this we perturb 

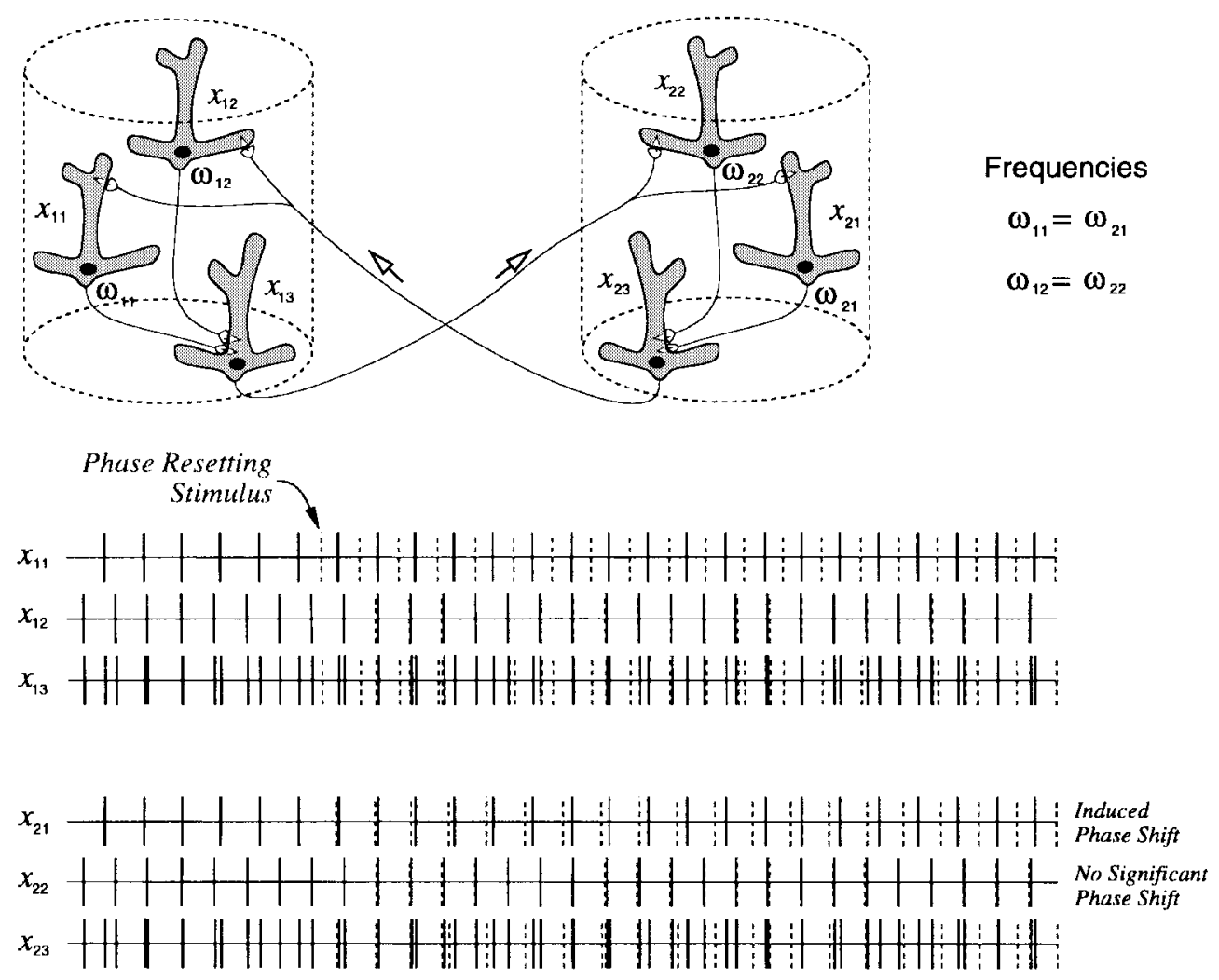

Fig. 3. Multiplexing of neural signals. Interaction between identical neurons $x_{11}$ and $x_{21}$ does not interfere with the interaction between the neurons $x_{12}$ and $x_{22}$ having different frequency even though all neurons use a single transmission line $x_{13}$. When we change the phase of $x_{11}$ by applying a phase resetting stimulus, the corresponding neuron $x_{21}$ acquires a phase shift, but $x_{22}$ does not (compare continuous and dashed bars). Parameters: Each column consists of strongly connected leaky integrate-and-fire neurons (2) with $b_{i j}=1, a_{11}=a_{21}=2, a_{21}=a_{22}=2.3$, and $a_{13}=a_{23}=0$. Connections between the columns $\varepsilon=1 / 20$ and time $t \in[0,20]$ (from [18]).

the phase of the first neuron without changing the phase of the second one. The old (new) activity is depicted as continuous (dashed) bars in Fig. 3. From the figure we can see that $x_{21}$ acquires a phase shift while $x_{22}$ does not. If we changed the phase of $x_{12}$, then $x_{21}$ would not acquire a phase shift but $x_{22}$ would. Thus, the pairs of neurons $x_{11}, x_{21}$ and $x_{12}, x_{22}$ oscillating with different frequencies can communicate selectively using different channels but a single transmission line. This is probably the simplest example of multiplexing of neural signals.

We see that the frequency of a periodically spiking neuron controls its communication with other neurons. We refer to such communication as being frequency modulated (FM interaction). In analogy with FM radio, we may say that the frequency (mean firing rate) of a periodically spiking neuron does not carry any information other than identifying a channel of communication. Information (i.e., neural code) is carried through modulations of interspike intervals, which are phase deviations $\varphi_{i}$. They are also referred to as being frequency modulations in electrical engineering literature.

Surprisingly, the result is much more general, and its applicability goes far beyond pulse-coupled neural networks. Hoppensteadt and Izhikevich [14, ch. 9] have proven this for all neural systems satisfying only two assumptions: 1) each unit is an autonomous oscillator and 2) the connections are weak. (If any of these assumptions is violated, then we can present counter-examples showing that FM interactions might not take place.) This result was extended later [18] to include oscillators having multifrequency autonomous rhythmic activity. In both cases each oscillator may describe dynamics of an excitable dendritic spine, a single cell, a cortical column, or entire brain structure, and consist of enormous number of variables and parameters taking into account all known (or still unknown) biophysical information. As soon as the two assumptions are satisfied, the interactions become FM.

The universality of FM interactions may shed some light on why the brain exhibits rhythmic activity, and why there are so many frequencies. We hypothesize [13] that neurons or cortical columns need rhythmic activity to communicate selectively. That is, they communicate only with those oscillators that have appropriate frequencies. They do not communicate with the other oscillators even though there might be synaptic connections between them. Thus, various ensembles of oscillators can process information without any cross-interference. An oscillator (a neuron or a cortical column) may participate in different ensembles simply by changing its frequency.

FM interactions might prove to be useful in design of neurocomputers, since they can avoid the $n^{2}$-connectivity problem. Indeed, a conventional neurocomputer having $n$ neurons must have at least $n(n-1) / 2$ connections [Fig. 4(a)], which makes building such a computer for large $n$ impractical. Now suppose that each neuron is a high-frequency oscillator 


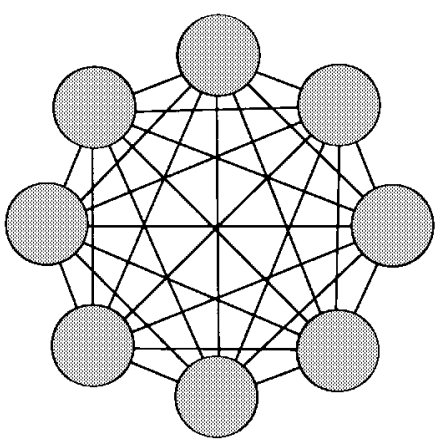

(a)

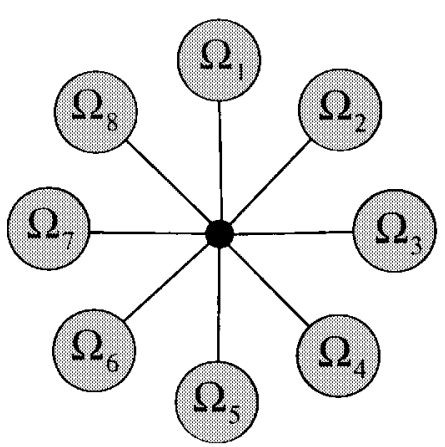

(b)

Fig. 4. (a) A conventional neurocomputer having $n$ neurons (shaded circles) would have at least $n(n-1) / 2$ connections and (b) an oscillatory neurocomputer can have only $n$ connections: from each neuron to a common media (black circle). The neurons can communicate selectively by changing the frequency, $\Omega_{i}$, of oscillation.

and the neurons communicate through a common medium [Fig. 4(b)] so that there are only $n$ connections. Then any two neurons can change dynamically the connection between them by changing their frequencies. In particular, they can turn the connections on and off. What we have proven here and in [13] and [14] is that this mechanism would work regardless of the technical details of how the neurons are connected, what their design is, etc. A potential problem is that whenever a neuron changes its frequency, it alters its connections with many other neurons too. It is not clear yet how to cope with this effect or to take advantage of it. In any case, programming such an oscillatory neurocomputer would require new concepts going beyond the Hopfield network paradigm.

\section{Synchronization: General THeORY}

Since weakly pulse-coupled oscillators can be dynamically partitioned into independent ensembles, it suffices to study information processing taking place within each such ensemble. Without loss of generality we assume that the entire network is a single ensemble; that is, we consider neurons having equal frequencies. Due to the phase model theorem, it suffices to consider the phase model (10).

The $i$ th and $j$ th neurons are said to be synchronized when

$$
\varphi_{j}(\tau)-\varphi_{i}(\tau) \rightarrow \chi_{i j}^{*}
$$

where $\chi_{i j}^{*}$ is some constant, and $\tau=\varepsilon t$ is the slow time. If $\chi_{i j}^{*}=0\left(\chi_{i j}^{*}=\pi\right)$, then synchronization is said to be
In-phase
Synchronization

Anti-phase
Synchronization

Out-of-phase Synchronization
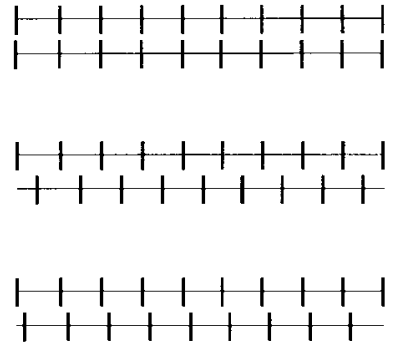

Fig. 5. Examples of various synchronization regimes.

in-phase (anti-phase). When $\chi_{i j}^{*}$ differs from zero and $\pi$, the synchronization is said to be out-of-phase; see Fig. 5 and [14, ch. 9] for more details. If every pair of neurons is synchronized, then so is the entire network. Obviously, it suffices to check that $\varphi_{1}$ is synchronized with $\varphi_{2}, \cdots, \varphi_{n}$.

In terms of the voltages $x_{i}$, the synchronization implies that $x_{i}$ and $x_{j}$ fire simultaneously. It does not imply, though, that $x_{i}(t) \approx x_{j}(t)$ for all $t$, unless $f_{i}(x) \approx f_{j}(x)$ for all $x$. This makes studying synchronization in (10) much easier than studying it in (1).

To find a synchronized solution in (10) one should find constants $\omega^{*}$ and $\varphi_{1}^{*}, \cdots, \varphi_{n}^{*}$, such that

$$
\omega^{*}=\omega_{i}+\sum_{j=1}^{n} H_{i j}\left(\chi_{i j}^{*}\right), \quad \text { for all } i
$$

where $\chi_{i j}^{*}=\varphi_{j}^{*}-\varphi_{i}^{*}$. Then the synchronized solution has the form

$$
\varphi_{i}(\tau)=\omega^{*} \tau+\varphi^{*}+\varphi_{i}^{*}, \quad i=1, \cdots, n
$$

where $\varphi^{*}$ is an arbitrary phase shift.

Let

$$
H_{i j}^{\prime}\left(\chi_{i j}^{*}\right)=\frac{d H_{i j}\left(\chi_{i j}^{*}\right)}{d \chi}
$$

denote the derivative of $H_{i j}$ at $\chi=\chi_{i j}^{*}$.

Theorem 2-Ermentrout 1992: The synchronized solution (12) is orbitally stable if all

$$
A_{i j}=H_{i j}^{\prime}\left(\chi_{i j}^{*}\right) \geq 0
$$

and the matrix $A=\left(A_{i j}\right)$ is irreducible; that is, the graph defined by $A$ is complete.

For example, when we consider a network of identical oscillators

$$
\varphi_{i}^{\prime}=\omega_{0}+\sum_{j=1}^{n} H_{0}\left(\varphi_{j}-\varphi_{i}\right)
$$

then the in-phase synchronized solution always exists, and it is stable when $H_{0}^{\prime}(0)>0$.

A generalization of the Ermentrout theorem for the case when the functions $H_{i j}$ are discontinuous at $\chi_{i j}^{*}$ is discussed in Section IV-B below.

The Ermentrout theorem provides only the sufficient condition for synchronization. Thus, synchronized regimes in (10) may be possible in other circumstances. 


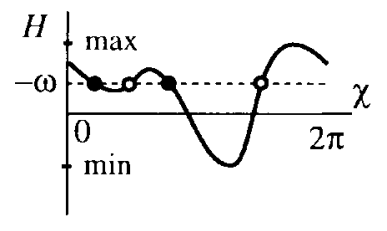

(a)

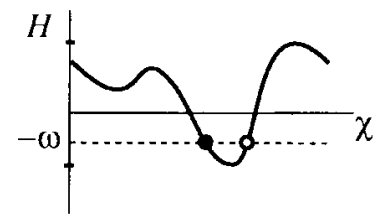

(b)

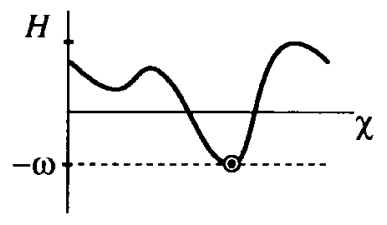

(c)

Fig. 6. Equilibria of (13) for various $\omega$.

\section{A. Example: Two Neurons}

It is usually difficult to find a solution to (11) when $n>2$. The case $n=2$ does not pose any problem, and can easily be analyzed. Let $\chi=\varphi_{2}-\varphi_{1}$ be the phase difference between two coupled neurons. Then

$$
\chi^{\prime}=\omega+H(\chi), \quad \chi \in \mathbb{S}^{1}
$$

where

$$
\omega=\omega_{2}-\omega_{1} \quad \text { and } \quad H(\chi)=H_{21}(-\chi)-H_{12}(\chi) .
$$

Some examples of $H$ are depicted in Fig. 9. Stable equilibria of (13) correspond to synchronized solutions of the phase model. Geometrically, the equilibria are the intersections of the graph of $H$ and the horizontal line $-\omega$, as we illustrate in Fig. 6. They are stable if the slope of the graph at the intersection is negative. (Do not mix this condition with the requirement of the Ermentrout theorem that derivatives of $H_{i j}$ are positive: $H$ and $H_{i j}$ are different, in some sense opposite functions.) When the parameter $\omega$ passes either $-\min H$ or - $\max H$, the synchronized solutions disappears via saddlenode bifurcation on a limit cycle [see Fig. 6(c)], and the new behavior is called drifting: The activity is either quasiperiodic or high order locked; see Chapter 9 by Hoppensteadt and Izhikevich [14] for detailed explanations.

\section{B. Discontinuous $H_{i j}$}

System (11) is usually easy to solve when functions $H_{i j}$ are discontinuous. Let $\gamma$ be a point of discontinuity of $H_{i j}$; that is

$$
a=\lim _{\chi \rightarrow \gamma-0} H_{i j}(\chi) \neq \lim _{\chi \rightarrow \gamma+0} H_{i j}(\chi)=b
$$

see left-hand side of Fig. 7. Whenever $\chi_{i j}^{*}=\gamma$ in (11), we allow $H_{i j}(\gamma)$ to assume any value between $a$ and $b$ so that the system is solved. The Ermentrout theorem can still be applied,
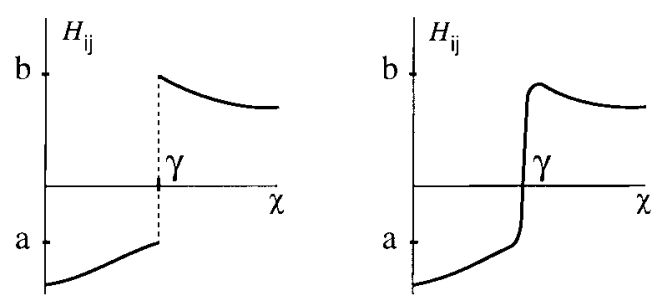

Fig. 7. A discontinuous function can be approximated by a continuous one, so that $H_{i j}(\gamma)$ can assume any value between $a$ and $b$.

but instead of looking at the sign of derivative at $\gamma$ to check the stability, we require that $a<b$.

Our motivation for this procedure is simple: We treat discontinuous functions as continuous ones in a singular limit; see right-hand side of Fig. 7. The inequality $a<b$ ensures that the derivative at $\gamma$ is positive.

Discontinuous functions $H_{i j}$ arise frequently in applications because the conditions $f_{i}(0)=f_{i}(1)$ and $g_{i j}(0)=g_{i j}(1)$ do not have to be satisfied in the pulse-coupled networks (1) even when $f_{i}$ and $g_{i j}$ are continuous. The resulting discontinuity of $H_{i j}(\chi)$ at $\chi=0$ is a mathematical consequence of the fact that pulse-coupled neural networks of the form (1) are ambiguous when two or more neurons fire simultaneously (see discussion in Appendix I-A). Surprisingly, the discontinuity leads to the following simple criterion for checking stability of in-phase synchronized solutions.

Theorem 3-Stability of In-Phase Synchronized Solutions: Consider pulse-coupled oscillators (1) having continuous functions $f_{i}(x)$ and $g_{i j}(x)$ at $x=0$ and $x=1$ and no transmission delays. If the in-phase synchronized solution exists, and

$$
\frac{g_{i j}(0)}{f_{i}(0)}<\frac{g_{i j}(1)}{f_{i}(1)}
$$

for all $i$ and $j$, then the solution is stable.

Since in-phase synchronized solutions (as well as antiphase solutions) always exist when the neurons are identical, one need only to check the condition (14) in this case. For example, the condition is satisfied for integrate-and-fire neurons, which implies that they synchronize in-phase.

Proof: We abuse notation below and use $\chi=0$ whenever $\chi \rightarrow 0+0$ and $\chi=2 \pi$ whenever $\chi \rightarrow 2 \pi-0$. From (8) and (14) it follows that $h_{i j}(2 \pi)>h_{i j}(0)$. Since there are no transmission delays, all $\psi_{i j}=0$, and from (7) it follows that

$$
H_{i j}(\chi)=\frac{1}{2 \pi} h_{i j}(-\chi)-h_{i j}^{0}
$$

and, hence, $a=H_{i j}(2 \pi)<H_{i j}(0)=b$.

Discontinuities in the connection functions $H_{i j}$ may result in the following phenomena:

- super-convergence to synchronized solution;

- perfect synchrony;

- persistence to heterogeneity.

The easiest way to see this is to consider a pair of oscillators and assume that the function $H$ in (13) has a discontinuity at $\gamma$ and $-\omega$ is between $a$ and $b$, as we depict in Fig. 8 . If $\chi(0)$ is in some neighborhood of $\gamma$, then it takes a finite amount of time to converge to it (i.e., super-convergence). 


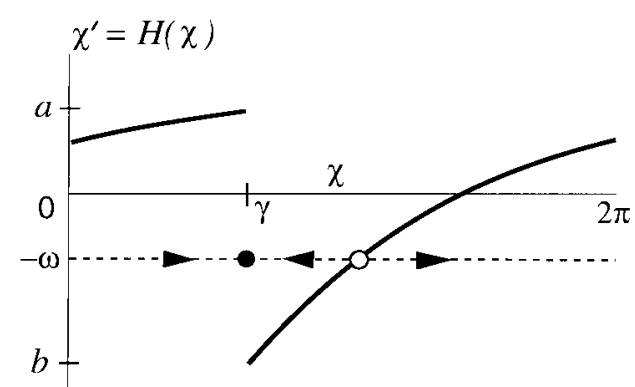

Fig. 8. Discontinuities in functions $H_{i j}$ or $H$ may lead to super-convergence to perfect synchrony and persistence to heterogeneity (see details in the text).

After the convergence the solution $\chi(\tau) \equiv \gamma$ for all $\tau$ (perfect synchrony). Moreover, $\chi$ stays at $\gamma$ even when we vary the parameter $\omega$ (i.e., persistence to heterogeneity). These properties of weakly pulse-coupled oscillators resemble those of relaxation oscillators [32].

A nasty drawback of discontinuous functions is that the solution may not be unique. Let us illustrate this issue using system (11). Suppose each $H_{i j}(\chi)$ has a discontinuity at $\chi=0$ (which is the case for integrate-and-fire neurons; see Section V). Since each $H_{i j}(0)$ assumes many values, then so does the parameter $\omega^{*}$ in the system

$$
\omega^{*}=\omega_{i}+\sum_{j=1}^{n} H_{i j}(0), \quad \text { for all } i
$$

which corresponds to in-phase synchronized solutions having different frequencies. Whatever frequency is observed in actual simulations of (1) depends on the algorithm used when two or more neurons fire simultaneously; see the Appendix I-A, for discussion of this issue.

\section{SYNCHRONIZATION: EXAMPLES}

Let us apply the theory described above to a number of well-known pulse-coupled networks.

\section{A. Integrate-and-Fire}

Integrate-and-fire neurons provide one of the simplest example of pulse-coupled networks [30]. Let $x_{i} \in[0,1]$ denote the rescaled membrane potential of the $i$ th neuron. A nonleaky integrate-and-fire neuron is governed by the equation

$$
\dot{x}_{i}=a_{i}
$$

until $x_{i}$ crosses the threshold value $x_{i}=1$ (the parameter $a_{i}$ must be positive to ensure that). At this moment, which we denote by $t_{i}^{*}$, it pulls membrane potentials of the other neurons by a fixed amount $\varepsilon$ possibly bringing them to the threshold too. Such system can be written in the form (1) where

$$
f_{i}\left(x_{i}\right)=a_{i} \quad \text { and } \quad g_{i j}\left(x_{i}\right)=1
$$

Simple calculations show that

$$
\begin{aligned}
T_{i} & =1 / a_{i} & & (\text { Period) } \\
\Omega_{i} & =2 \pi a_{i} & & \text { (Frequency) } \\
X_{i}(t) & =a_{i} t & & \text { (The Solution) } \\
H_{i j}(\chi) & =0 & & {[\text { see }(10)] } \\
H(\chi) & =0 & & {[\text { see }(13)] }
\end{aligned}
$$

see the top part of Fig. 9. Since $H_{i j} \equiv 0$, we see that such a pulse-coupled network behaves as if it were uncoupled on the time scale of order $1 / \varepsilon$; that is, the distance between successive firings of the $i$ th and the $j$ th neurons does not change with time; see illustration in Fig. 10. Behavior of such a neural network is not interesting even when we introduce inhibitory synapses or explicit synaptic transmission delays.

\section{B. Leaky Integrate-and-Fire}

A network of leaky integrate-and-fire neurons is described by the system

$$
\dot{x}_{i}=a_{i}-b_{i} x_{i}+\varepsilon \sum_{j=1}^{n} g_{i j} \delta\left(t-t_{j}^{*}\right)
$$

where we require that $a_{i}>b_{i}>0$ so that each neuron is an autonomous oscillator. Peskin [30], Mirollo and Strogatz [28], Kuramoto [20], and many others have studied synchronization phenomena in such a network.

1) Excitatory Connections: Suppose all $g_{i j}=1$. It is a simple exercise to check that

$$
\begin{aligned}
T_{i} & =-\frac{1}{b_{i}} \ln \left(1-\frac{b_{i}}{a_{i}}\right) \\
\Omega_{i} & =-\frac{2 \pi b_{i}}{\ln \left(1-\frac{b_{i}}{a_{i}}\right)} \\
X_{i}(t) & =\frac{a_{i}}{b_{i}}\left(1-e^{-b_{i} t}\right) \\
H_{i j}(\chi) & =\frac{\Omega_{i} \Omega_{j}}{2 \pi a_{i}}\left(1-\frac{b_{i}}{a_{i}}\right)^{\theta_{i} / 2 \pi}-\frac{\Omega_{i}^{2} \Omega_{j}}{2 \pi a_{i}\left(a_{i}-b_{i}\right)} \\
H(\chi) & =\frac{\Omega^{2}}{2 \pi a}\left\{\left(1-\frac{b}{a}\right)^{-\theta_{i} / 2 \pi}-\left(1-\frac{b}{a}\right)^{\theta_{i} / 2 \pi}\right\} .
\end{aligned}
$$

See Fig. 9 for illustrations. Notice that each $H_{i j}(\chi)$ is discontinuous at $\chi=0$, and

$$
\lim _{\chi \rightarrow 2 \pi-0} H_{i j}(\chi)<\lim _{\chi \rightarrow 0+0} H_{i j}(\chi) .
$$

This results in super-convergence to the in-phase synchronized solution, which we illustrate in Fig. 11. Moreover, the solution persists under small heterogeneity of the frequencies; see Section IV-B. Mirollo and Strogatz [28] and Kuramoto [20] obtained the same result using different methods.

Analysis of the shape of the functions $H_{i j}$ can say more about behavior of the integrate-and-fire neurons. For example, the fact that the derivative of $H_{i j}$ is negative implies that even if a completely out-of-phase synchronized solution existed, it would be unstable. (Indeed, it follows from the Ermentrout 

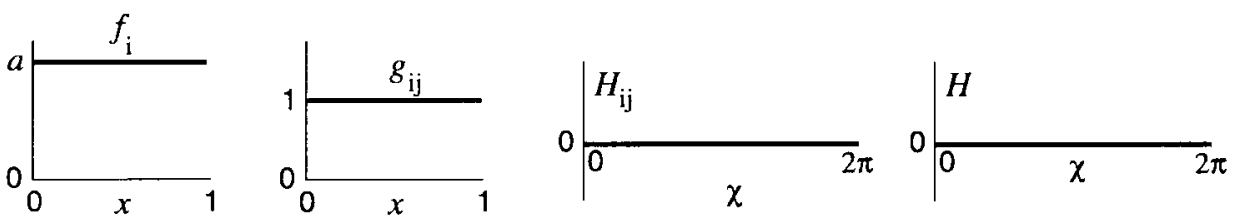

\section{Integrate-and-Fire}
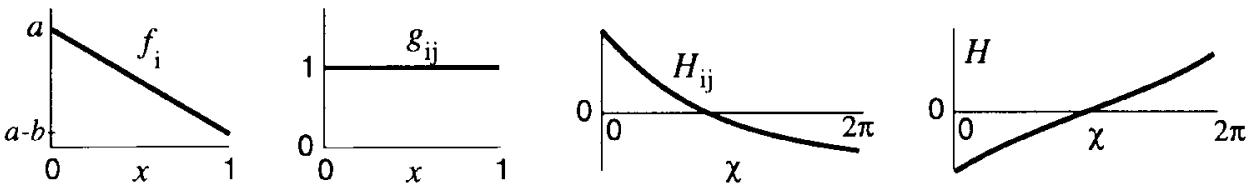

Leaky Integrate-and-Fire
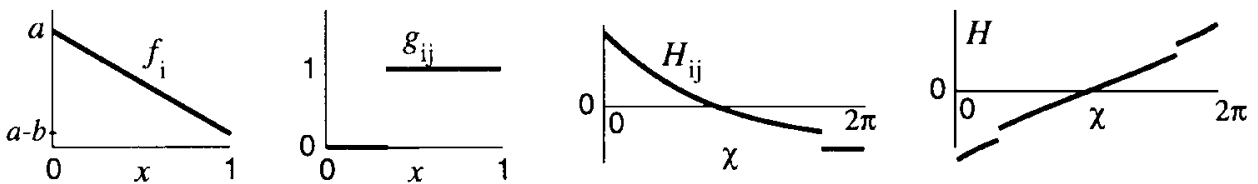

Leaky Integrate-and-Fire with Absolute Refractory Period

Fig. 9. Various functions for integrate-and-fire neurons.

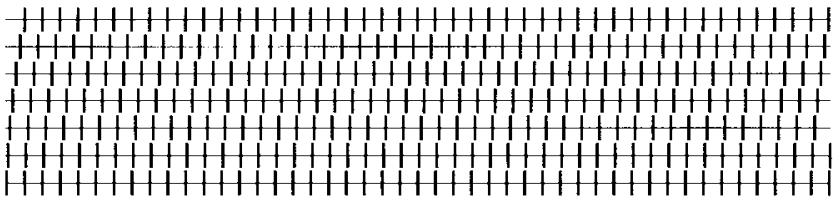

Fig. 10. Simulation of $n=7$ nonleaky integrate-and-fire neurons The network behaves as if the neurons were uncoupled. (Parameters: $a_{i}=1, b_{i}=0, \varepsilon=1 / 20, t \in[0,30]$.)

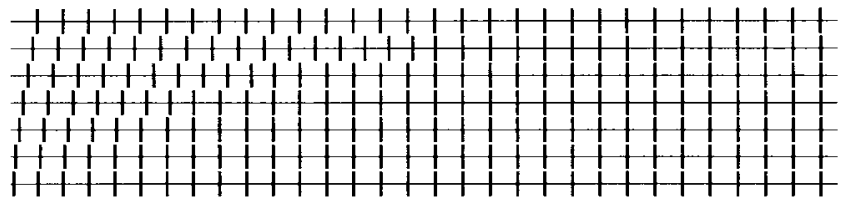

Fig. 11. Super-convergence to the in-phase synchronized solution in a network of $n=7$ integrate-and-fire neurons having instantaneous excitatory connections. (Parameters: $a_{i}=1, b_{i}=1 / 2, \varepsilon=1 / 20, t \in[0,30]$.)

theorem that any such solution would be stable for $t \rightarrow-\infty$.) Thus, a stable splay (merry-go-around) state cannot exist in such a network.

Now suppose there is an explicit time delay $\eta_{i j}$, which induces a phase shift in connection functions $H_{i j}$, as we depict in Fig. 12. From the Ermentrout theorem it follows that the in-phase synchronized solution becomes unstable in this case since each $H_{i j}^{\prime}(0)<0$. But many other stable solutions may appear (see illustration in Fig. 13) including the asynchronous states [1]. This is in agreement with the results obtained by Coombes and Lord [3].

2) Inhibitory Connections: Suppose the synaptic connections are inhibitory; that is, all $g_{i j}<0$. Each connection function $H_{i j}$ looks like the one depicted in Fig. 14(a). The

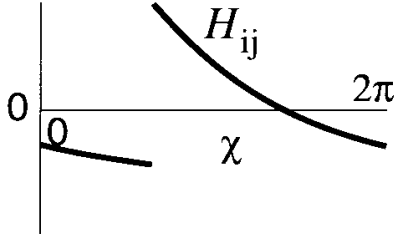

Fig. 12. Explicit synaptic transmission delay (e.g., 1/3 of a period) induces a phase shift $(2 \pi / 3)$ in the connection function $H_{i j}$ corresponding to the leaky integrate-and-fire neuron.

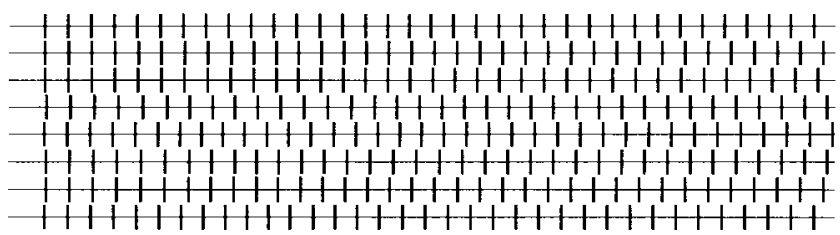

Fig. 13. Desynchronization of the integrate-and-fire network with an explicit transmission delay. In-phase synchronization is not a stable solution. (Parameters: $n=8, a_{i}=1, b_{i}=1 / 2, \eta=1 / 3, \varepsilon=1 / 20, t \in[0,30]$.)

in-phase synchronized solution continues to exist but becomes unstable. Since $H_{i j}^{\prime}(\chi)>0$ for all $\chi \neq 0$, any antiphase or out-of-phase synchronized solution, if exists, becomes stable. This confirms the result of Nishura et al. [29].

Let us introduce a small synaptic transmission delay. Then each $H_{i j}$ acquires a phase shift and look qualitatively similar to the one depicted in Fig. 14(b). Since $H_{i j}^{\prime}(0)>0$ in this case, the in-phase synchronized solution, if exists, becomes stable (see Fig. 15), but there is no super-convergence, perfect synchrony, and persistence to heterogeneity. Since in-phase solution always exists in the case of two identical neurons, we confirm the result of Van Vreeswijk and coauthors [34] 


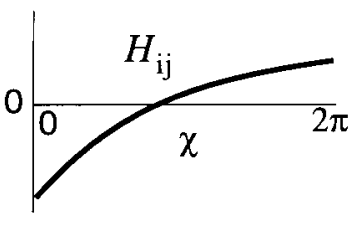

(a)

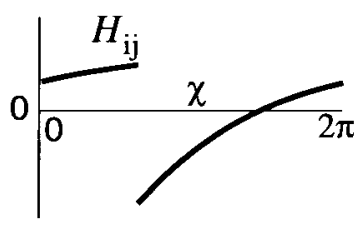

(b)

Fig. 14. (a) Function $H_{i j}$ for integrate-and-fire neurons with inhibitory synapses and (b) the same function is phase-shifted by $2 \pi / 3$ due to the synaptic transmission delay that takes a third of a cycle.

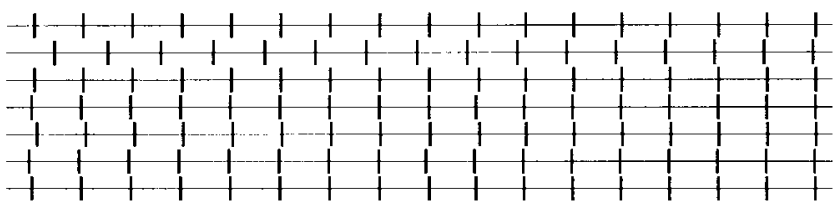

Fig. 15. Convergence to the in-phase synchronized solution in the inhibitory integrate-and-fire network with explicit transmission delays. (Parameters: $a_{i}=1, b_{i}=1 / 2, \eta=1 / 3, \varepsilon=1 / 20, t \in[0,30]$.)

that inhibition, not excitation, may synchronize neural firing of two identical neurons having noninstantaneous synaptic transmission.

3) What Matters in Neural Locking: Let us summarize what we found so far. We study effect of excitation, inhibition, and transmission delay on the dynamics of leaky integrate-and-fire neurons. We confirm results of other authors (who used different methods) that neither of these factors alone contributes to the in-phase synchronization, but their combination does. For example, the in-phase state is superstable for excitatory instantaneous connections, but becomes unstable or disappears when transmission delay is introduced provided that the delay is not proportional to the period. In contrast, the in-phase synchronized state is unstable when the connections are inhibitory, but introduction of a transmission delay can make it stable.

\section{Leaky Integrate-and-Fire with Refractory Period}

An obvious modification of the integrate-and-fire neuron is an introduction of the absolute refractory period during which the cell is not sensitive to any input. This results in piecewise constant function $g_{i j}$ that is zero for all $x_{i}$ from some neighborhood of $x_{i}=0$; see bottom of Fig. 9. One can modify the integrate-and-fire neurons further and further to take into account more and more biophysical information. This would result in a pulse-coupled model that would be as complex and cumbersome as the Hodgkin-Huxley neurons.

\section{Class 1-Excitable Oscillators}

Let us ask the following question: "Are there any neural network models that are as simple as the integrate-and-fire
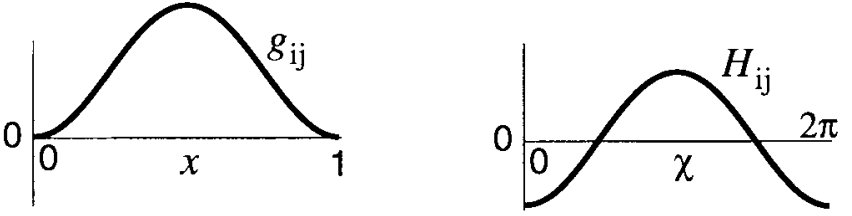

Fig. 16. Pulse-coupled canonical model (15) for Class 1 neural excitability.

model, yet as biologically plausible as Hodgkin-Huxley-type models?" Remarkably, there are. More precisely, there are pulse-coupled canonical models that possess the following universal property: many biophysically detailed and biologically plausible Hodgkin-Huxley-type neural networks can be transformed into the pulse-coupled canonical models by a piece-wise continuous change of variables [19]. Thus, the only difference between the pulse-coupled canonical models and the biophysically detailed neural models is just a matter of coordinate change. The only major requirement is that the biophysically detailed models exhibit Class 1 neural excitability [11]; that is, they can fire with an arbitrary low frequency. As a result, the question of biological plausibility of such canonical models is replaced by the question of biological plausibility of Class 1 neural excitability. This is the essence of the canonical model approach developed in the book by Hoppensteadt and Izhikevich [14].

Derivation of such pulse-coupled canonical models can be found in [14] and [19]. It is based on the mathematical techniques developed by Ermentrout and Kopell [4], [7] to study parabolic bursting. The pulse-coupled canonical model that corresponds to periodic spiking of identical neurons (see [19]) can be written in the form (1)

$$
\dot{x}_{i}=1+\varepsilon\left(1-\cos 2 \pi x_{i}\right) \sum_{j=1}^{n} s_{i j} \delta\left(x_{j}\right) .
$$

The shape of the connection function $g_{i j}\left(x_{i}\right)=1-\cos 2 \pi x_{i}$ is depicted in Fig. 16. Notice that it is continuous and $g_{i j}(0)=0$, which can be interpreted as follows: The neurons are not sensitive to external inputs when they are in a process of generating an action potential. Thus, such $g_{i j}$ takes into account absolute and relative refractory periods. It is amazing that such $g_{i j}$ was not "postulated," "invented," or "motivated by biology," but was obtained as a result of the Ermentrout-Kopell change of variables applied to an arbitrary Class 1 excitable system.

System (15) can be transformed into the phase model (10). It has the form

$$
\varphi_{i}^{\prime}=\omega_{i}+\sum_{j=1}^{n} H_{i j}\left(\varphi_{j}-\varphi_{i}\right), \quad H_{i j}(\chi)=-2 \pi s_{i j} \cos \chi
$$

where

$$
\omega_{i}=2 \pi \sum_{j=1}^{n} s_{i j}
$$

Notice that $H_{i j}$, which is depicted in Fig. 16, can be represented as $H_{i j}(\chi)=2 \pi s_{i j} \sin (-\pi / 2+\chi)$. When (15) has transmission delays, then there is an induced phase shift added 
to $-\pi / 2$. In any case, the canonical model can be written in the Kuramoto form [21]

$$
\varphi_{i}^{\prime}=\omega_{i}+\sum_{j=1}^{n} s_{i j} \sin \left(\psi_{i j}+\varphi_{j}-\varphi_{i}\right)
$$

where we incorporated $2 \pi$ into $s_{i j}$ and $-\pi / 2$ into $\psi_{i j}$. Thus, the Kuramoto model is canonical for Class 1 excitable oscillators having delayed interactions.

Let us study the existence and stability of various synchronized solutions in (16). Notice that each $H_{i j}$ is an even function. As a result, a network of two identical oscillators can maintain an arbitrary phase shift because $H_{12}=H_{21}$ and

$$
\chi^{\prime}=H(\chi)=H_{21}(-\chi)-H_{12}(\chi)=0
$$

for all $\chi \in \mathbb{S}^{1}$; see Section IV-A. This agrees with the result obtained in [19]. Since each $H_{i j}^{\prime}(0)=0$, the in-phase synchronized solution cannot be exponentially stable. In fact, it is unstable when $n>2$ (see [19]). Both results confirm and extend numerical [10] and analytical [4] findings that difficulty to synchronize is a natural property of Class 1 excitable oscillators that is relatively independent from the equations that describe each cell. In this sense Class 1 excitable neurons are quite different from the integrate-and-fire neurons.

As has been pointed out before [4], behavior of identical Class 1 excitable oscillators is not generic in the sense that small heterogeneity (different $\omega_{i}$ and/or $s_{i j}$ ) or synaptic transmission delay (phase shift of $H_{i j}$ ) can change it significantly. For example, in Section VII-D below we show that the Class 1 excitable oscillators can learn and reproduce the in-phase synchronized state (as well as any other temporal pattern) by modifying the transmission delays.

\section{Oscillatory Associative MEMORY: General TheORY}

Hoppensteadt and Izhikevich [14, Th. 9.15] have proven the following result, which is an analog of Cohen-Grossberg convergence theorem for nonoscillatory neurons:

Theorem 4-Convergence Theorem for Oscillatory Neural Networks: Consider the phase model (10) and suppose that $\omega_{1}=\cdots=\omega_{n}=\omega$ and

$$
H_{i j}(-\chi)=-H_{j i}(\chi), \quad \chi \in \mathbb{S}^{1}
$$

for all $i, j=1, \cdots, n$. Then the network dynamics converge to a limit cycle. On the limit cycle, all neurons oscillate with equal frequencies and constant phase deviations. This corresponds to synchronization of the network activity.

Since there can be many such limit cycles, the phase model (10) and hence the pulse-coupled network (1) can act as a multiple attractor type neural network; see Fig. 17. Whether or not this property renders any advantages over the classical Hopfield network (where attractors are just equilibria) is still an open question.

The convergence theorem can be illustrated using the $\mathrm{Ku}$ ramoto model (17). The requirement (18) implies that

$$
s_{i j}=s_{j i} \quad \text { and } \quad \psi_{i j}=-\psi_{j i}
$$

for all $i$ and $j$. A learning rule that satisfies this requirement is discussed later.

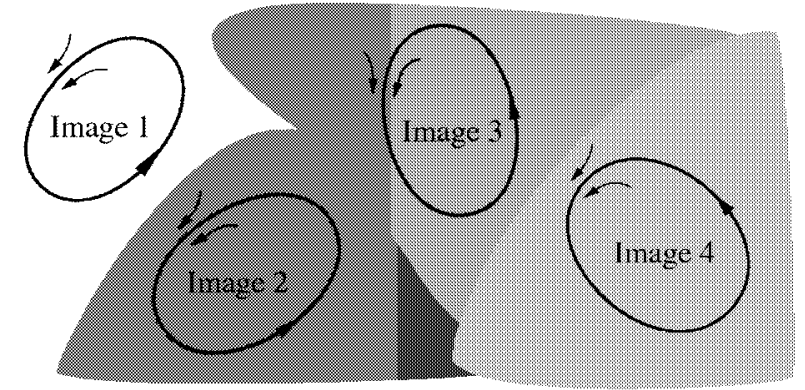

Fig. 17. Each memorized image is represented as a limit cycle attractor in the phase space of the model (from [14]).

\section{A. Integrate-and-Fire}

It is easy to see that condition (18) cannot be satisfied for $H_{i j}$ corresponding to the integrate-and-fire networks considered above unless all $H_{i j}=H_{j i}=0$. This does not imply, though, that such networks have trivial associative properties because the convergence theorem provides only a sufficient condition. Below we modify the integrate-and-fire network so that we can apply the convergence theorem and guarantee that integrate-and-fire-type networks have oscillatory associative memory. Moreover, we show that there can be many such modifications.

\section{B. Weakly Pulse-Coupled Oscillators}

Below we consider the following pulse-coupled network:

$$
\dot{x}_{i}=1+\varepsilon \sum_{j=1}^{n} g_{i j}\left(x_{i}\right) \delta\left(t-t_{j}^{*}-\eta_{i j}\right)
$$

where we take $f_{i}=1$ so that each oscillator has period $T=1$ and frequency $\Omega=2 \pi$. We do not lose any generality, because we prove a lemma in the Appendix that shows that nonconstant $f_{i}$ could be transformed into constant ones by a continuous change of variables.

Theorem 5-The Convergence Theorem For Weakly Pulse-Coupled Oscillators: Consider the weakly pulsecoupled network (19) and the corresponding phase model (10). Let $g(x)$ be an arbitrary odd function having period 1 , see examples in Fig. 18. Let $g_{i j}^{0}, g_{j i}^{0}, w_{i j}$, and $w_{j i}$ be arbitrary constants. If the connection functions have the form

$$
\begin{aligned}
& g_{i j}\left(x_{i}\right)=g_{i j}^{0}+g\left(w_{i j}+x_{i}\right) \\
& g_{j i}\left(x_{j}\right)=g_{j i}^{0}+g\left(w_{j i}+x_{j}\right)
\end{aligned}
$$

and

$$
\eta_{i j}+w_{i j}+\eta_{j i}+w_{j i}=0 \quad(\bmod 1)
$$

where $(\bmod 1)$ means modulo 1 . Then the condition $(18)$ in the convergence theorem for oscillatory neural networks is satisfied. If, in addition

$$
\sum_{j=1}^{n} g_{i j}^{0}=\sum_{j=1}^{n} g_{k j}^{0}
$$

for all $i$ and $k$, then the activity of the pulse-coupled network (19) converges to an $\varepsilon$-neighborhood of a limit cycle. On the 

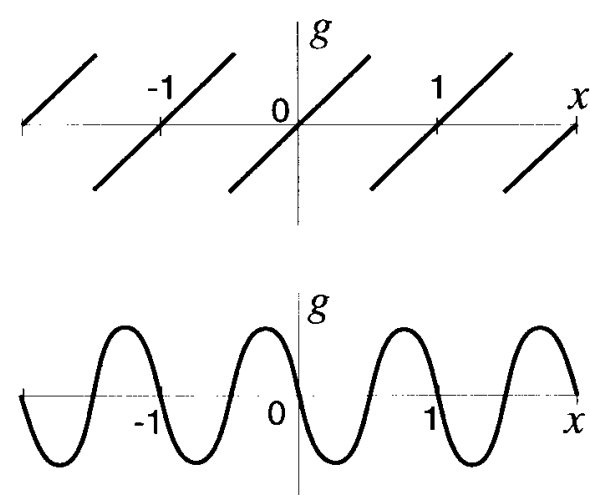

Fig. 18. Examples of odd functions having period 1 (see Theorem 5 for details).

limit cycle all neurons fire with equal frequencies and constant phase differences, which corresponds to synchronization of the network activity.

Since there may be many such limit cycles, each for each memorized image, the pulse-coupled network (19) can act as a multiple attractor neural network provided that $\varepsilon$ is not very large. The network can store and retrieve oscillatory images in essentially the same way the Hopfield network does with static images; see examples in Fig. 20.

Proof: From (8) it follows that

$$
\begin{aligned}
h_{i j}(\theta) & =4 \pi^{2} g_{i j}\left(w_{i j}+\frac{\theta}{2 \pi}\right) \\
& =4 \pi^{2}\left\{g_{i j}^{0}+g\left(\frac{2 \pi w_{i j}+\theta}{2 \pi}\right)\right\} .
\end{aligned}
$$

Since $g$ is odd, it has zero average, and from (9) it follows that

$$
h_{i j}^{0}=2 \pi g_{i j}^{0} .
$$

From (7) it follows that

$$
\begin{aligned}
H_{i j}(\chi) & =\frac{1}{2 \pi} h_{i j}\left(\psi_{i j}-\chi\right)-2 \pi g_{i j}^{0} \\
& =2 \pi g\left(\frac{2 \pi w_{i j}+\psi_{i j}-\chi}{2 \pi}\right)
\end{aligned}
$$

where $\psi_{i j}=2 \pi \eta_{i j}(\bmod 2 \pi)$ is the phase shift that is due to the transmission delay $\eta_{i j}$. Obviously

$$
\begin{aligned}
H_{i j}(-\chi) & =2 \pi g\left(\frac{2 \pi w_{i j}+\psi_{i j}+\chi}{2 \pi}\right) \\
& =-2 \pi g\left(\frac{-2 \pi w_{i j}-\psi_{i j}-\chi}{2 \pi}\right) \quad(g \text { is odd }) \\
& =-2 \pi g\left(\frac{2 \pi w_{j i}+\psi_{j i}-\chi}{2 \pi}\right) \\
& =-H_{j i}(\chi)
\end{aligned}
$$

which completes the proof of the first part of the theorem.

Equation (23) implies that $\omega_{i}=\omega_{k}$ for all $i$ and $k$. The rest follows from the phase model Theorem 1 and the convergence Theorem 5 .

It should be stressed that the theorem above provides only the sufficient condition for the pulse-coupled neural network
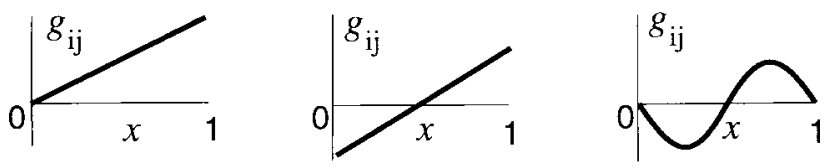

Fig. 19. Functions $g_{i j}$ corresponding to the pulse-coupled systems (24)-(26), respectively.

(19) to have oscillatory associative memory. Thus, other pulsecoupled networks that do not satisfy the assumptions of the theorem should not be discarded immediately without an additional analysis.

\section{OSCILLATORY AsSOCIATIVE MEMORY: EXAMPLES}

\section{A. Instantaneous Synaptic Transmission}

Let us consider the case when there are no transmission delays; that is, when all $\eta_{i j}=0$. We start from the following question: "Are there any simple pulse-coupled neural networks that are guaranteed to have oscillatory associative memory?" Below is a list of some of them

$$
\begin{aligned}
& \dot{x}_{i}=1+\varepsilon x_{i} \sum_{j=1}^{n} s_{i j} \delta\left(x_{j}\right) \\
& \dot{x}_{i}=1+\varepsilon\left(x_{i}-0.5\right) \sum_{j=1}^{n} s_{i j} \delta\left(x_{j}\right) \\
& \dot{x}_{i}=1-\varepsilon \sin 2 \pi x_{i} \sum_{j=1}^{n} s_{i j} \delta\left(x_{j}\right)
\end{aligned}
$$

where we used $x_{j}(t)$ instead of $t-t_{j}^{*}$ because $x_{j}(t)=t+\mathcal{O}(\varepsilon)$ when it fires a spike. The corresponding functions $g_{i j}$ are depicted in Fig. 19.

Each model has its own advantages and drawbacks. For example, the seemingly simplest function $g_{i j}\left(x_{i}\right)=s_{i j} x_{i}$ in (24) has a nonzero average $g_{i j}^{0}=s_{i j} / 2$. Therefore, the condition (23) has the form

$$
\sum_{j=1}^{n} s_{i j}=\text { const } \quad \text { (independent of } i \text { ) }
$$

and can be interpreted as follows: All neurons in the network have equal amount of "postsynaptic sites." The condition may easily be violated during learning period unless a special learning rule is used.

System (25) is free from this drawback, but the function $g_{i j}\left(x_{i}\right)=s_{i j}\left(x_{i}-0.5\right)$ changes its sign. This may seem to be biologically implausible if one interprets positive $g_{i j}$ as "excitation" and negative $g_{i j}$ as "inhibition." However, there is another interpretation: Negative $g_{i j}$ delays the next firing of the ith neuron, while positive $g_{i j}$ advances it. Whenever $g_{i j}$ changes signs implies that the effect of firing of the $j$ th neuron onto the $i$ th neuron depends on the relative timing of their firing. If $s_{i j}>0$ in the system (25), and $x_{i}$ just fired $\left(x_{i}<0.5\right)$, then a firing of $x_{j}$ delays the next firing of $x_{i}$. In contrast, if $x_{i}$ is about to fire a spike $\left(x_{i}>0.5\right)$, then firing of the $j$ th neuron advances this event. This occurs in many biophysically detailed Hodgkin-Huxley-type models. 

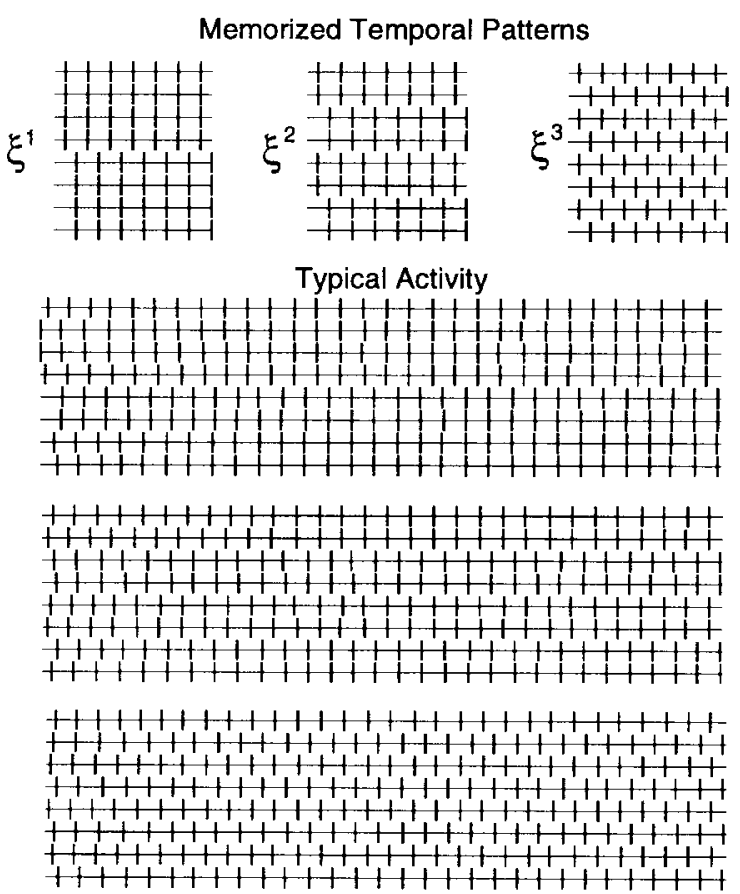

$g(x)=x-0.5$
Memorized Temporal Patterns
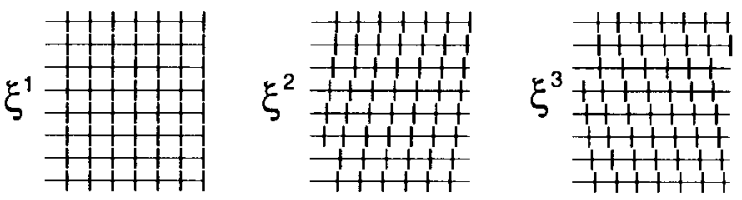

Typical Activity
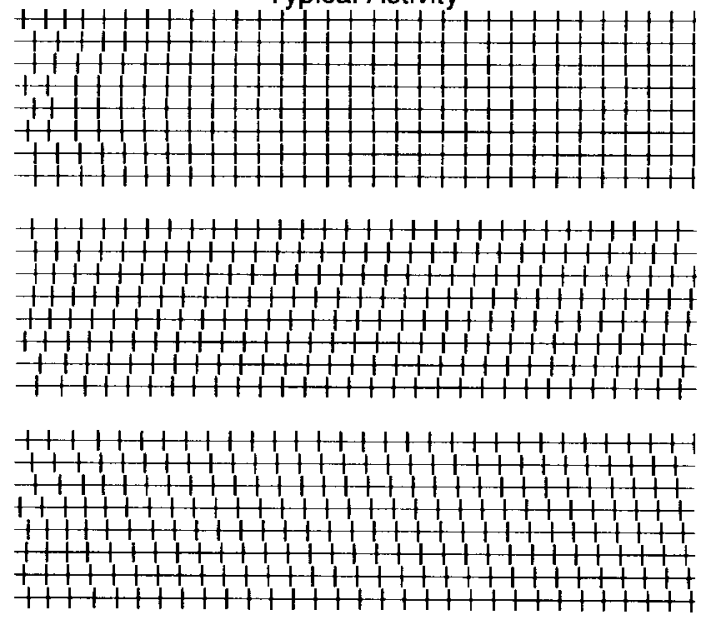

$g(x)=-\sin 2 \pi x$

Fig. 20. A pulse-coupled neural network (30) can memorize temporal patterns (in-phase, anti-phase, and out-of-phase synchronized solutions) by modifying synaptic weights $s_{i j}$ and transmission delays $\eta_{i j}$. Parameters: $\varepsilon=1 / 20, n=8, t \in[0,30]$.

System (26) exhibits essentially the same behavior as (25), but the connection function $g_{i j}\left(x_{i}\right)=-s_{i j} \sin 2 \pi x_{i}$ is continuous and $g_{i j}(0)=g_{i j}(1)=0$. Such network does not have nasty problems associated with discontinuities, such as nonuniqueness of solutions; see Section IV-B. Moreover, we find (but cannot explain) that this model learns out-of-phase temporal patterns better than (25) does; see Fig. 20.

\section{B. Learning Rule}

Let us discuss possible learning rules for the pulse-coupled networks for which the convergence theorem above is applicable. To be as general as possible, we consider the networks in the following form:

$$
\dot{x}_{i}=1+\varepsilon \sum_{j=1}^{n} s_{i j} g\left(w_{i j}+x_{i}\right) \delta\left(x_{j}\right)
$$

where $g(x)$ is an odd function having period " 1 ." Without loss of generality we assume that it changes signs from "+" to "-" when $x$ passes $x=0$; see examples in Fig. 19. We use $g_{i j}^{0}=0$ so that the condition (23) is always satisfied regardless of the values of $s_{i j}$. The convergence Theorem 5 requires that

$$
w_{i j}=-w_{j i}(\bmod 1) \quad \text { and } \quad s_{i j}=s_{j i} .
$$

It is convenient to introduce a complex synaptic coefficient

$$
c_{i j}=s_{i j} e^{2 \pi i w_{i j}} .
$$

Then, the requirement above means that the complex synaptic matrix $C=\left(c_{i j}\right)$ is self-adjoint; i.e.,

$$
c_{i j}=\bar{c}_{j i}
$$

for all $i$ and $j$. Notice, that this condition is general and does not depend on form of the odd function $g$.

The requirement that $C$ is self-adjoint arises naturally in weakly connected networks near multiple Andronov-Hopf bifurcations [14]-[16], for which a learning rule is well known: Let the complex variable

$$
z_{i}=e^{2 \pi \mathbf{i} x_{i}}
$$

denote the periodic activity of the $i$ th neuron (the neuron fires when $z_{i}$ crosses the positive part of the real line $\mathbb{R}$ ). The learning rule [14], [16] has the following simple form:

$$
\dot{c}_{i j}=\alpha z_{i} \bar{z}_{j}-\gamma c_{i j}
$$

where $\alpha$ and $\gamma$ are some (small) positive parameters. The latter has the meaning of the fading constant. Since we do not have any better idea, we take the learning rule (29) and apply it to pulse-coupled oscillators (28).

Suppose $x_{i}$ and $x_{j}$ fire incoherently. Then the product $z_{i} \bar{z}_{j}$ assumes many values randomly, and from the averaging it follows that $c_{i j} \rightarrow 0$, which implies that the connections between incoherently firing neurons weaken.

Now suppose that $x_{i}$ and $x_{j}$ fire periodically with a constant phase shift. For example, $x_{i}$ fires $t_{i j}$ units of time after $x_{j}$ does; that is

$$
x_{i}(t)=x_{j}\left(t-t_{i j}\right)(\bmod 1) .
$$

Due to the special form of (28), we have

$$
x_{j}\left(t-t_{i j}\right)=x_{j}(t)-t_{i j}+\mathcal{O}(\varepsilon)(\bmod 1)
$$


and hence

$$
x_{i}(t)-x_{j}(t)=-t_{i j}+\mathcal{O}(\varepsilon)(\bmod 1)
$$

for all $t$. From

$$
\dot{c}_{i j}=\alpha e^{-2 \pi i t_{i j}}-\gamma c_{i j}
$$

it follows that

$$
c_{i j} \rightarrow \frac{\alpha}{\gamma} e^{-2 \pi i t_{i j}}
$$

where we neglected the small term $\mathcal{O}(\varepsilon)$. We see that

$$
s_{i j} \rightarrow \frac{\alpha}{\gamma} \quad \text { and } \quad w_{i j} \rightarrow-t_{i j}(\bmod 1) .
$$

The former limit implies that the synaptic connection $s_{i j}$ strengthens. Let us show that the latter limit implies that the neurons learn the phase shift $t_{i j}$. That is, whenever $x_{j}$ fires, $x_{i}$ tends to fire with the memorized time delay $t_{i j}$. Indeed, suppose $w_{i j}=-t_{i j}$, then there can be three cases.

- $x_{i}$ fires too early. This happens when $x_{i}(t)>x_{j}\left(t-t_{i j}\right)$. At the moment $x_{j}$ fires, $x_{i}(t)-t_{i j}=x_{i}(t)+w_{i j}>0$, hence $g\left(x_{i}+w_{i j}\right)<0$, which causes a delay of the firing of the $i$ th neuron.

- $x_{i}$ fires too late. Hence $x_{i}(t)<x_{j}\left(t-t_{i j}\right)$, hence $x_{i}(t)+w_{i j}<0$ and $g\left(x_{i}+w_{i j}\right)>0$, which causes an advance in the firing of the $i$ th neuron.

- The neurons fire with exact time difference $t_{i j}$. Then $g_{i j}\left(x_{i}+w_{i j}\right)=0$, and the difference is preserved.

Thus, the learning rule (29) memorizes not only correlation between the firings of the neurons, but also the phase information; that is, the relative timing of their firings.

One may claim that (29) is not biologically plausible because it requires the knowledge of the presynaptic membrane potential $x_{j}$ at all times. An obvious modification of (29), which uses only the timing of the presynaptic spiking, has the following form:

$$
\dot{c}_{i j}=\alpha e^{2 \pi \mathrm{i} x_{i}} \delta\left(x_{j}\right)-\gamma c_{i j}
$$

Here $c_{i j}$ is incremented by $\alpha e^{2 \pi \mathbf{i} x_{i}}$ whenever the $j$ th neuron fires. Since such firings occur every $1+\mathcal{O}(\varepsilon)$ units of time, we can rewrite the equation above in the following form:

$$
c_{i j}^{\text {new }}=\alpha e^{2 \pi i x_{i}}+e^{-1} \gamma c_{i j}^{\text {old }}
$$

where the reset from $c_{i j}^{\text {old }}$ to $c_{i j}^{\text {new }}$ occurs when $x_{j}$ fires.

\section{Transmission Delays: Timing Is Everything}

Consider the pulse-coupled system (28) with nonzero transmission delay constants $\eta_{i j}$

$$
\dot{x}_{i}=1+\varepsilon \sum_{j=1}^{n} s_{i j} g\left(w_{i j}+x_{i}\right) \delta\left(x_{j}\left(t-\eta_{i j}\right)\right) .
$$

The condition (22) implies that in order to have oscillatory associative memory, the transmission delays $\eta_{i j}$ and $\eta_{j i}$ must be in a certain relation with the parameters $w_{i j}$ and $w_{j i}$.
When the delays are nonzero and fixed, the learning rule may have the form

$$
\dot{c}_{i j}=\alpha_{i j} z_{i} \bar{z}_{j}-\gamma c_{i j}
$$

where the complex-valued parameters

$$
\alpha_{i j}=\alpha e^{-2 \pi \mathrm{i} \eta_{i j}} \quad \text { and } \quad \alpha_{j i}=\alpha e^{-2 \pi \mathrm{i} \eta_{j i}}
$$

compensate for the delays. Such learning rule allows the network to memorize the phase information in the same way it does if there were no delays (as we describe in the Section VI).

It is also feasible to consider the case when the parameters $w_{i j}$ and $w_{j i}$ are fixed, but the transmission delays $\eta_{i j}$ and $\eta_{j i}$ can be modified due to the learning. Let

$$
c_{i j}=s_{i j} e^{-2 \pi i \eta_{i j}}
$$

be the complex-valued synaptic coefficient (notice the minus sign). The learning rule has the form (31), but

$$
\alpha_{i j}=\alpha e^{2 \pi \mathbf{i} w_{i j}} \quad \text { and } \quad \alpha_{j i}=\alpha e^{2 \pi \mathrm{i} w_{j i}}
$$

compensate for the phase shift in $g$. For example, when $w_{i j}=w_{j i}=0$, the parameters $\alpha_{i j}$ and $\alpha_{j i}$ are real, and the rule produces the delays satisfying

$$
\eta_{i j}+\eta_{j i}=0(\bmod 1)
$$

We interpret this as follows: It takes precisely one period (or a multiple of a period) for a spike to travel from the $j$ th neuron to the $i$ th neuron and back to the $j$ th one.

There can be many other learning rules for the pulse-coupled network (30) so that it has an oscillatory associative memory. A seemingly counterintuitive fact is that one can "teach" the network by modifying only the synaptic transmission delays $\eta_{i j}$ without modifying the synaptic efficacy $s_{i j}$. This emphasizes the importance of spike timing code as opposed to mean firing rate code and puts a new twist into the idea of "syn-fire" chains [2].

Example-Learning Temporal Patterns: To illustrate the idea of learning via modifying the synaptic connections and the transmission delays, we consider the pulse-coupled system (30) for $g(x)=x-0.5$ and for $g(x)=-\sin 2 \pi x$. This is the same as to take the systems (25) and (26) with transmission delays. Each synchronized temporal pattern can be represented as a complex vector

$$
\xi=\left(\begin{array}{c}
1 \\
e^{2 \pi \mathrm{i} y_{2}} \\
\vdots \\
e^{2 \pi \mathrm{i} y_{n 2}}
\end{array}\right) \in \mathbb{C}^{n}
$$

and can be interpreted as follows: When neuron $x_{1}$ fires a spike $\left(x_{1}=1\right.$, therefore $\left.e^{2 \pi \mathbf{i} x_{1}}=1\right)$, the second neuron has membrane potential $x_{2}=y_{2}$, etc. Obviously, the network activity is synchronized (possibly out-of-phase) in this case.

Let $\xi^{1}, \cdots, \xi^{m}$ be the set of temporal patterns to be memorized; see illustration at the top of Fig. 20. We take the complex synaptic matrix in the form $C$

$$
C=\sum_{s=1}^{m} \xi^{s}\left(\xi^{s}\right)^{\top}
$$


where $T$ means transpose and complex-conjugate. Each coefficient

$$
c_{i j}=\sum_{s=1}^{m} \xi_{i}^{s} \bar{\xi}_{j}^{s}
$$

is easily computed, and the synaptic weight $s_{i j}=\left|c_{i j}\right|$ and the delay $\eta_{i j}=\operatorname{Arg} c_{i j}$ can easily be determined. The latter is defined only when $s_{i j} \neq 0$. Notice the similarity with the Hebbian rule, in which all $\xi_{i}^{s}$ are real and, hence, there is no need for conjugation. We set $s_{i i}=0$ for all $i$ in our simulations in Fig. 20.

From the figure we can see that each key patter $\xi^{s}$ builds a periodic attractor in the phase space of the network. If the initial condition is chosen near the attractor, then the network converge to it. Notice that the convergence takes just a few spikes, which is far below the theoretical limit of $1 / \varepsilon=20$ spikes.

\section{Class 1 Excitable Oscillators}

Let us apply the convergence theorem for pulse-coupled networks to the canonical model for Class 1 excitable neurons having delayed interactions. Such a model can be written in the form

$$
\dot{x}_{i}=1+\varepsilon\left(1-\cos 2 \pi x_{i}\right) \sum_{j=1}^{n} s_{i j} \delta\left(x_{j}\left(t-\eta_{i j}\right)\right) .
$$

The function $g_{i j}\left(x_{i}\right)=s_{i j}\left(1-\cos 2 \pi x_{i}\right)$ can be represented in the form (20) when

$$
g_{i j}^{0}=s_{i j}, \quad g(x)=s_{i j} \sin 2 \pi x, \quad w_{i j}=-1 / 4 .
$$

The condition (22) has the form

$$
\eta_{i j}+\eta_{j i}=1 / 2(\bmod 1)
$$

and has an obvious interpretation: It takes half a cycle (plus a multiple of the period) for a spike to travel from the $j$ th neuron to the $i$ th one and back to the $j$ th. If, in addition, $s_{i j}=s_{j i}$ for all $i$ and $j$, and (27) holds, then the pulse-coupled canonical model has an oscillatory associative memory. Again, one can "teach" the strength of synaptic connections $s_{i j}$ and/or the synaptic transmission delays $\eta_{i j}$ to memorize oscillatory images.

We stress that whether or not the pulse-coupled network (32) has any advantage over the classical Hopfield network is still an open question. This is especially frustrating after we take into account that (32) is a canonical model for all weakly coupled Class 1 excitable oscillators regardless of the equations that describe dynamics of each cell [19].

\section{DISCUSSION}

The major purpose of this paper is to explore the link between the theory of pulse-coupled neural networks and the theory of weakly connected oscillators. Our major tool is Theorem 1 according to which all weakly pulse-coupled oscillatory networks of the form (1) can be converted into a phase model of the form (6) by a piece-wise continuous change of variables. Since the only difference between the former and the latter is just a matter of coordinate change, we study the phase model and deduce many interesting facts about behavior of pulse-coupled networks.

\section{A. FM Interactions}

Each variable $\varphi_{i}$ in the phase model (6) has the meaning of timing of firing of the $i$ th neuron. Each function $H_{i j}$ describes how timing of spiking of the $j$ th neuron affects that of the $i$ th one on the time scale of order $1 / \varepsilon$. When the neurons have nonresonant frequencies, the corresponding function $H_{i j} \equiv 0$ even when $x_{i}$ and $x_{j}$ are connected in the original pulsecoupled network (1). This means that such connections are functionally insignificant and do not affect dynamics of the neurons. (They may contribute though to a slight increase or decrease of the mean firing rate, since they affect the parameter $\omega_{i}$.)

We see that whether or not two weakly pulse-coupled neurons interact depends not only on the existence of connections between them, but also on the frequencies of their firing. In analogy with FM radio, we say that connections between such neurons are frequency modulated (FM). In particular, we conclude that the mean firing rate (the frequency) of a periodically spiking neuron does not carry any information other than identifying a channel of communication. The information (neural code) is carried via modulations of interspike intervals.

\section{B. Synchronization}

To study the existence and stability of synchronized solutions in the pulse-coupled model one should solve the algebraic equation (11) and apply the Ermentrout theorem. This relatively easy procedure provides a powerful tool for analysis of pulse-coupled networks. In particular, it allows us to confirm and extend results of others (see Section V) regarding the locking dynamics of integrate-and-fire neurons: Neither the sign of synaptic connections (i.e., excitation or inhibition), nor the synaptic transmission delays alone contribute to synchronization, but their combination does. We also find that behavior of integrate-and-fire neurons may be quite different from the behavior of other pulse-coupled networks.

\section{Oscillatory Associative Memory}

One of the most important contributions of phase models into the theory of pulse-coupled networks is the convergence theorem for oscillatory neural networks; see Section VI. The theorem provides a criterion for checking the existence of oscillatory associative memory in pulse-coupled networks. Moreover, it provides an algorithm for invention of pulsecoupled networks that are guaranteed to have associative properties. Such pulse-coupled networks can memorize and reproduce temporal patterns in the same way a standard Hopfield network does with stationary patterns. Learning in such pulse-coupled networks may consist in modifying not only the weights of synaptic connections, but also the duration of synaptic transmission delays. 


\section{Limitations}

Our results are exact when the strength of synaptic connections is not very large; that is, when $\varepsilon \leq \varepsilon_{0}$, where $\varepsilon_{0}>0$ is some parameter that depends on the details of the network. In particular, almost all illustrations in this paper were made for $\varepsilon=1 / 20$. To which extent the results are valid for large $\varepsilon$ is an open question. We hope however that they can still provide an adequate intuition into the behavior of strongly pulse-coupled neural networks.

\section{APPENDIX I}

\section{Proof of the Phase Model THeOrem For WEAKLY PULSE-COUPLED OSCILLATORS}

Let us prove the following general lemma, which can be interpreted as follows: Any "leaky" pulse-coupled oscillatory network can be transformed into "nonleaky" form by an appropriate continuous change of variables.

Lemma 6: There is a continuous change of variables that transforms (1) into

$$
\dot{\theta}_{i}=\Omega_{i}+\varepsilon \sum_{j=1}^{n} h_{i j}\left(\theta_{i}\right) \delta\left(\theta_{j}\left(t-\eta_{i j}\right)\right)+\mathcal{O}\left(\varepsilon^{2}\right)
$$

where each $h_{i j}$ is the phase resetting curve defined below (35). Firing a spike corresponds to $\theta_{j}$ crossing $2 \pi \equiv 0$. At this moment it increments $\theta_{i}$ by $\varepsilon h_{i j}\left(\theta_{i}\right) \Omega_{j}^{-1}$.

The factor $\Omega_{j}^{-1}$ is due to the fact that $\theta_{j}(t)$ behaves like $\Omega_{j} t$ when it crosses 0 , and

$$
\int \delta\left(\theta_{j}(t)\right) d t=\int \delta\left(\Omega_{j} t\right) d t=\frac{1}{\Omega_{j}} \int \delta(s) d s=\frac{1}{\Omega_{j}} .
$$

Proof: Let $X_{i}(t), t \in\left[0, T_{i}\right]$, be a solution of (3) starting from $X_{i}(0)=0$. We represent it in the form

$$
X_{i}(t)=X_{i}\left(\Omega_{i}^{-1} \theta_{i}(t)\right) \text {. }
$$

Since

$$
1=X_{i}\left(T_{i}\right)=X_{i}\left(\Omega_{i}^{-1} 2 \pi\right)
$$

the firing of the $i$ th neuron occurs when $\theta_{i}=2 \pi$.

When neurons do not fire (i.e., when all $x_{j} \neq 1$ ), we differentiate both sides of (34) with respect to $t$ and divide by $f_{i}>0$ to obtain

$$
\dot{\theta}_{i}=\Omega_{i}
$$

When the $j$ th neuron fires, $x_{j}=1$, and $X_{i}$ is incremented by $\varepsilon g_{i j}\left(X_{i}\right)$. At this moment $\theta_{j}$ crosses $2 \pi \equiv 0$. Since it behaves like $\Omega_{j} t+\mathcal{O}(\varepsilon)$, it increments the phase variable $\theta_{i}$ by $\varepsilon \Omega_{j}^{-1} h_{i j}\left(\theta_{i}\right)$. The function $h_{i j}$ can be determined from

$$
\begin{aligned}
X_{i}+\varepsilon g_{i j} & =X_{i}\left(\Omega_{i}^{-1}\left(\theta_{i}+\varepsilon \Omega_{j}^{-1} h_{i j}+\mathcal{O}\left(\varepsilon^{2}\right)\right)\right) \\
& =X_{i}+\varepsilon f_{i}\left(X_{i}\right)\left(\Omega_{i} \Omega_{j}\right)^{-1} h_{i j}+\mathcal{O}\left(\varepsilon^{2}\right) .
\end{aligned}
$$

This leads to

$$
h_{i j}\left(\theta_{i}\right)=\Omega_{i} \Omega_{j} \frac{g_{i j}\left(X_{i}\left(\Omega_{i}^{-1} \theta_{i}\right)\right)}{f_{i}\left(X_{i}\left(\Omega_{i}^{-1} \theta_{i}\right)\right)}
$$

which completes the proof of the lemma.
Even though the difference between (1) and (33) is a matter of coordinate change, the latter may have certain advantages: It has fewer nonlinear terms. Hence, it may be easier to analyze and/or simulate.

\section{A. Possible Discontinuity of $h_{i j}$}

Each function $h_{i j}(\theta)$ may be discontinuous at $\theta=0$ when $f_{i}(0) \neq f_{i}(1)$ or $g_{i j}(0) \neq g_{i j}(1)$. Such a discontinuity, which affects behavior of the system only when two or more neurons fire simultaneously, reflects the ambiguity of the pulse-coupled system (1) when neurons fire simultaneously.

Let us illustrate this issue when $g_{i j}(0) \neq g_{i j}(1)$ and $x_{i}$ and $x_{j}$ fire simultaneously. In practice they never fire simultaneously, so we have $x_{i} \approx x_{j}$ at the moment of firing. Suppose $x_{i}$ fires first. Whether it advances or delays $x_{j}$ is irrelevant, because the spike from the $j$ th neuron arrives when $x_{i}$ has already crossed $x_{i}=1$. The increment of $x_{i}$ is

$$
\varepsilon g_{i j}(0)+\mathcal{O}\left(\varepsilon^{2}\right)
$$

in this case. In contrast, if $x_{j}$ fires first, it increments $x_{i}$ by $\varepsilon g_{i j}(1)+\mathcal{O}\left(\varepsilon^{2}\right)$, which is different from (36). Therefore, it is not clear what value we should use if they do fire simultaneously. The apparent contradiction is reflected in discontinuities of $h_{i j}$ and $H_{i j}$ in Theorem 1.

\section{B. Phase Deviation Variables}

Let us represent each $\theta_{i}$ in the form

$$
\theta_{i}(t)=\Omega_{i} t+\phi_{i}
$$

where $\phi_{i} \in \mathbb{S}^{1}$ is the phase deviation from the natural oscillation $\Omega_{i} t$. It accounts for modulations of the interspike intervals that are due to the weak inputs from the other neurons. To determine the phase $\theta_{i}$, and hence the voltage $x_{i}$ of the $i$ th neuron, it suffices to determine its phase deviation $\phi_{i}$. Knowing phase deviations provides complete information about behavior of the network.

We rewrite the phase model (33) in terms of phase deviations $\phi_{i}$. For this we substitute (37) into (33) to obtain

$\dot{\phi}_{i}=\varepsilon \sum_{j=1}^{n} h_{i j}\left(\Omega_{i} t+\phi_{i}\right) \delta\left(\Omega_{j}\left(t-\eta_{i j}\right)+\phi_{j}\left(t-\eta_{i j}\right)\right)+\mathcal{O}\left(\varepsilon^{2}\right)$.

Averaging theory [14, Sec. 9.3] provides a nearly identical change of variables (valid for all $\varepsilon$ less than certain $\varepsilon_{0}>0$ )

$$
\phi_{i}=\varphi_{i}+\mathcal{O}(\varepsilon)
$$

that transforms this system into the form

$$
\varphi_{i}^{\prime}=\sum_{j=1}^{n} \bar{h}_{i j}\left(\varphi_{i}, \varphi_{j}\right)+\mathcal{O}(\varepsilon)
$$

where $^{\prime}=d / d \tau, \tau=\varepsilon t$ is the slow time, and $\bar{h}_{i j}\left(\varphi_{i}, \varphi_{j}\right)$ is the "average" of the term $h_{i j} \delta$ given by

$$
\lim _{T \rightarrow \infty} \frac{1}{T} \int_{0}^{T} h_{i j}\left(\Omega_{i} t+\varphi_{i}\right) \delta\left(\Omega_{j}\left(t-\eta_{i j}\right)+\varphi_{j}\left(t-\eta_{i j}\right)\right) d t .
$$

It depends not only on the form of $h_{i j}$, but also on the resonant relations between the frequencies $\Omega_{i}$ and $\Omega_{j}$. 


\section{Equal Frequencies}

Let us consider the simplest case when $\Omega_{i}=\Omega_{j}=\Omega_{0}$ for some $\Omega_{0}$. (The case $\Omega_{i} \approx \Omega_{j}$ is considered in Section I-F below.) Let $T_{0}=2 \pi / \Omega_{0}$ be the period of oscillation. Then (40) converges to the integral

$$
\begin{aligned}
\bar{h}_{i j} & =\frac{1}{T_{0}} \int_{0}^{T_{0}} h_{i j}\left(\Omega_{0} t+\varphi_{i}\right) \delta\left(\Omega_{0}\left(t-\eta_{i j}\right)+\varphi_{j}\right) d t \\
& =\frac{1}{2 \pi} \int_{0}^{2 \pi} h_{i j}\left(s+\Omega_{0} \eta_{i j}+\varphi_{i}-\varphi_{j}\right) \delta(s) d s \\
& =\frac{1}{2 \pi} h_{i j}\left(\Omega_{0} \eta_{i j}+\varphi_{i}-\varphi_{j}\right)
\end{aligned}
$$

where $\varphi_{j}=\varphi_{j}\left(t-\eta_{i j}\right)$, and we do not change the integration limits because $h_{i j}$ is $2 \pi$-periodic. Thus, the system (39) can be written in the form

$$
\dot{\varphi}_{i}=\frac{\varepsilon}{2 \pi} \sum_{j=1}^{n} h_{i j}\left(\psi_{i j}+\varphi_{i}-\varphi_{j}\left(t-\eta_{i j}\right)\right)+\mathcal{O}\left(\varepsilon^{2}\right)
$$

where $\psi_{i j}=\Omega_{0} \eta_{i j}$ is the rescaled transmission delay. Let $\tau=\varepsilon t$ be the slow time. Then

$$
\varphi_{j}\left(\varepsilon\left(t-\eta_{i j}\right)\right)=\varphi_{j}\left(\tau-\varepsilon \eta_{i j}\right)=\varphi_{j}(\tau)+\mathcal{O}(\varepsilon)
$$

and we rewrite the system above in the form

$$
\varphi_{i}^{\prime}=\frac{1}{2 \pi} \sum_{j=1}^{n} h_{i j}\left(\psi_{i j}+\varphi_{i}(\tau)-\varphi_{j}(\tau)\right)+\mathcal{O}(\varepsilon) .
$$

Here we implicitly assumed that the delay constants, $\eta_{i j}$, are of the same order of magnitude as the periods $T_{i}$. In this case the delay affects only the small term $\mathcal{O}(\varepsilon)$ in the equation above. This seems to be a universal principle in the theory of weakly connected oscillators [5], [14]. In contrast, if the delay is long enough and comparable with $1 / \varepsilon$ periods, then the averaged equation above does acquire an explicit time delay [17].

\section{Resonant Frequencies}

We say that the frequencies $\Omega_{i}$ and $\Omega_{j}$ are resonant if

$$
p \Omega_{j}=q \Omega_{i}
$$

for some relatively prime integers $p$ and $q$. One can prove an analog of [14, Th. 9.6 or 9.12] to show that the average $\bar{h}_{i j}$ can be represented in the form

$$
\bar{h}_{i j}\left(\varphi_{i}, \varphi_{j}\right)=P_{i j}\left(p \varphi_{j}-q \varphi_{i}\right)
$$

for some $2 \pi$-periodic function $P_{i j}$, which approaches the constant $h_{i j}^{0}$ defined below when the order of the resonance $|p+q|$ increases.

Indeed, since $p T_{i}=q T_{j}$, (40) converges to the integral

$$
\begin{aligned}
\bar{h}_{i j} & =\frac{1}{q T_{j}} \int_{0}^{q T_{j}} h_{i j}\left(\Omega_{i} t+\varphi_{i}\right) \delta\left(\Omega_{j}\left(t-\eta_{i j}\right)+\varphi_{j}\right) d t \\
& =\frac{1}{2 \pi q} \int_{0}^{2 \pi q} h_{i j}\left(\frac{p}{q} s+\frac{p}{q} \eta_{i j}+\varphi_{i}-\frac{p}{q} \varphi_{j}\right) \delta(s) d s \\
& =\frac{1}{2 \pi q} \sum_{k=0}^{q-1} h_{i j}\left(\frac{p}{q} 2 \pi k+\frac{p}{q} \eta_{i j}+\varphi_{i}-\frac{p}{q} \varphi_{j}\right) .
\end{aligned}
$$

Let us check that the sum is a $2 \pi / q$-periodic function of $\varphi_{i}$. For this notice that $p$ and $q$ are relatively prime, therefore, the set

$$
\left\{0, \frac{2 \pi p}{q}(\bmod 2 \pi), \cdots,(q-1) \frac{2 \pi p}{q}(\bmod 2 \pi)\right\}
$$

is a permutation of the set

$$
\left\{0, \frac{2 \pi}{q}, 2 \frac{2 \pi}{q}, \cdots,(q-1) \frac{2 \pi}{q}\right\}
$$

Hence, the sum can be rearranged in the form

$$
\frac{1}{2 \pi q} \sum_{k^{\prime}=0}^{q-1} h_{i j}\left(\frac{2 \pi}{q} k^{\prime}+\frac{p}{q} \eta_{i j}+\varphi_{i}-\frac{p}{q} \varphi_{j}\right)=Q\left(\varphi_{i}-\frac{p}{q} \varphi_{j}\right)
$$

where $Q$ is some $2 \pi / q$-periodic function. Let $P_{i j}(y)=$ $Q(y / q)$, then $P_{i j}$ is $2 \pi$-periodic, and (41) holds.

If we treat $P_{i j}$ (or $Q$ ) as the rectangular method approximation to the integral of $h_{i j}$ with the integration step $2 \pi / q$, then we can conclude that

$$
\bar{h}_{i j}=P_{i j}=h_{i j}^{0}+\mathcal{O}\left(\frac{1}{q}\right)
$$

that is, $P_{i j}$ approaches a constant when the order of the resonance increases.

\section{E. Nonresonant Frequencies}

We say that the frequencies $\Omega_{i}$ and $\Omega_{j}$ are nonresonant if

$$
p \Omega_{j} \neq q \Omega_{i}
$$

for any nonzero integers $p$ and $q$. In this case the integral (40) converges to the constant

$$
h_{i j}^{0}=\frac{1}{(2 \pi)^{2}} \int_{0}^{2 \pi} h_{i j}(s) d s .
$$

The proof follows from the ergodic theory and is provided elsewhere. Intuitively, it also follows from (42) when $q \rightarrow \infty$.

Finally, we can write the averaged phase model in the form (6), where

$$
H_{i j}\left(\varphi_{i}, \varphi_{j}\right)=\bar{h}_{i j}\left(\varphi_{i}, \varphi_{j}\right)-h_{i j}^{0}
$$

is a connection function having zero mean value, and

$$
\omega_{i}=\sum_{j=1}^{n} h_{i j}^{0}
$$

From (34), (37), and (38) it follows that

$$
x_{i}(t)=X_{i}\left(t+\Omega_{0}^{-1} \varphi_{i}(\varepsilon t)\right)+\mathcal{O}(\varepsilon) .
$$

We depicted typical $x_{i}(t)$ and $X_{i}\left(t+\Omega_{0}^{-1} \varphi_{i}(\varepsilon t)\right)$ in Fig. 1 . 


\section{F. Nearly Equal Frequencies}

We can relax the requirement from Section I-C and assume that all

$$
\Omega_{i}=\Omega_{0}+\varepsilon \beta_{i}
$$

where $\beta_{i}$ are frequency deviations. Thus, we consider a weakly heterogeneous network. Applying transformations (37) and (38) as if all $\beta_{i}$ were zeros, we get

$$
\varphi_{i}^{\prime}=\omega_{i}+\beta_{i}+\frac{1}{2 \pi} \sum_{j=1}^{n} H_{i j}\left(\varphi_{i}, \varphi_{j}\right)+\mathcal{O}(\varepsilon) .
$$

This system can be written in the form (6) if we incorporate $\beta_{i}$ into $\omega_{i}$.

\section{APPENDIX II}

\section{GENERALIZATION: TEMPORAL SYNAPTIC TRANSMISSION}

The phase model theorem 1 can easily be extended to pulse-coupled networks having nontrivial temporal synaptic transmission. Below we summarize the theory for nearly identical neurons. Our exposition is based on [34].

If neurons experience firings of other neurons as brief increments or decrements of their membrane potentials, then we can use Dirac delta functions and write the pulse-coupled system in the form (1). In contrast, when the postsynaptic effect is extended in time (e.g., it has slow rise), then the pulse-coupled network should be written in the form

$$
\dot{x}_{i}=f\left(x_{i}\right)+\varepsilon \sum_{j=1}^{n} g_{i j}\left(x_{i}\right) E_{j}\left(t-t_{j}^{*}\right)
$$

where $t_{j}^{*}$ denotes the time of last spiking of the $j$ th neuron, and $E_{j}(t)$ is some function that describes the temporal effect of synaptic transmission. For example, $E_{j}$ can be the alpha function

$$
E_{j}(s)=\alpha^{2} s e^{-\alpha s}, \quad \alpha>0, \quad s=t-t_{j}^{*} \geq 0
$$

describing the postsynaptic conductance. Corresponding $g_{i j}$ has the form

$$
g_{i j}\left(x_{i}\right)=x_{\mathrm{syn}}-x_{i}
$$

where $x_{\text {syn }} \in \mathbb{R}$ is some constant. It is commonly assumed that $E_{j}(s) \equiv 0$ when $s$ is greater than the period of the $j$ th oscillator, which means that the secretion of synaptic transmitter due to the incoming spike is much greater than that due to the previous spikes.

In general, each $x_{i}$ is a vector describing the membrane potential, ion conductances, and other electro-physiological characteristics of neurons. In this case one should use the Malkin theorem [14, Th. 9.2] to convert (43) into the phase model (10).

When all $x_{i}$ are scalars, and the neurons have nearly identical frequencies $\Omega_{i}=\Omega_{j}=\Omega_{0}$, then the phase model theorem 1 can be proved [34], but the functions $h_{i j}(\theta)$ defined in (8) have the following form

$$
h_{i j}(\theta)=\Omega_{0}^{2} \int_{0}^{T_{0}} \frac{g_{i j}\left(X_{i}(t)\right)}{f_{i}\left(X_{i}(t)\right)} E_{j}\left(t+\Omega_{0}^{-1} \theta\right) d t
$$

where $T_{0}=2 \pi / \Omega_{0}$ is the common period.

\section{ACKNOWLEDGMENT}

The author would like to acknowledge F. Hoppensteadt whose collaboration and constant support have been invaluable during the last five years. Y.-T. (Jim) Wang read the manuscripts and made a number of useful suggestions.

\section{REFERENCES}

[1] L. F. Abott and C. V. Vreeswijk, "Asynchronous states in networks of pulse-coupled oscillators," Phys. Rev. E, vol. 48, pp. 1483-1490.

[2] M. Abeles, Corticonics: Neural Circuits of the Cerebral Cortex. New York: Cambridge Univ. Press, 1991.

[3] S. Coombes and G. J. Lord, "Intrinsic modulation of pulse-coupled integrate-and-fire neurons," Phys. Rev. E, vol. 56, pp. 5809-5818, 1997.

[4] G. B. Ermentrout, "Type I membranes, phase resetting curves, and synchrony," Neural Comput., vol. 8, pp. 979-1001, 1996.

[5] "An introduction to neural oscillators," in Neural Modeling and Neural Networks, F. Ventriglia, Ed. Oxford, U.K.: Pergamon, pp. 79-110, 1994.

[6] _ , "Stable periodic solutions to discrete and continuum arrays of weakly coupled nonlinear oscillators," SIAM J. Appl. Math., vol. 52, pp. 1665-1687, 1992.

[7] G. B. Ermentrout and N. Kopell, "Parabolic bursting in an excitable system coupled with a slow oscillation," SIAM J. App. Math., vol. 46, pp. 233-253, 1986

[8] U. Ernst, K. Pawelzik, and T. Geisel, "Synchronization induced by temporal delays in pulse-coupled oscillators," Phys. Rev. Lett., vol. 74, pp. 1570-1573, 1995.

[9] W. Gerstner, "Time structure of the activity in neural network models," Phys. Rev. E, vol. 51, pp. 738-758, 1995.

[10] D. Hansel, G. Mato, and C. Meunier, "Synchrony in excitatory neural networks," Neural Comput., vol. 7, pp. 307-335, 1995.

[11] A. L. Hodgkin, "The local electric changes associated with repetitive action in a nonmedulated axon," J. Physiol., vol. 107, pp. 165-181, 1948.

[12] J. J. Hopfield and A. V. M. Herz, "Rapid local synchronization of action potentials: Toward computation with coupled integrate-and-fire neurons," in Proc. Nat. Academy Sci. USA, 1995, vol. 92, pp. 6655-6662.

[13] F. C. Hoppensteadt and E. M. Izhikevich, "Thalamo-cortical interactions modeled by weakly connected oscillators: Could brain use FM radio principles?" BioSystems, 1998, accepted.

[14] _ Weakly Connected Neural Networks. New York: SpringerVerlag, 1997.

[15] _ "Synaptic organizations and dynamical properties of weakly connected neural oscillators-I: Analysis of canonical model," Biol. Cybern., vol. 75, pp. 117-127, 1996.

[16] , "Synaptic organizations and dynamical properties of weakly connected neural oscillators-II: Learning of phase information," Biol. Cybern., vol. 75, pp. 129-135, 1996.

[17] E. M. Izhikevich, "Phase models with explicit time delays," Phys. Rev. $E$, submitted.

[18] -Weakly connected quasiperiodic oscillators, FM interactions, and multiplexing in the brain," SIAM J. Appl. Math., 1998, accepted.

[19] _ "Class 1 neural excitability, conventional synapses, weakly connected networks, and mathematical foundations of pulse-coupled models," this issue, pp. 499-507.

[20] Y. Kuramoto, "Collective synchronization of pulse-coupled oscillators and excitable units," Phys. D, vol. 50, pp. 15-30, 1991.

[21] Chemical Oscillations, Waves, and Turbulence. New York: Springer-Verlag, 1984

[22] T. Lindblad and J. M. Kinser, Image Processing Using Pulse-Coupled Neural Networks. London, U.K.: Springer-Verlag, 1997.

[23] W. Maass, "Networks of spiking neurons: The third generation of neural network models," Neural Networks, vol. 10, pp. 1659-1671, 1997.

[24] A. Mason, A. Nicoll, and K. Stratford, "Synaptic transmission between individual pyramidal neurons of the rat visual cortex, in vitro," The Journal of Neuroscience, vol. 11, pp. 72-84, 1991.

[25] R. Mathar and J. Mattfeldt, "Pulse-coupled decentral synchronization," SIAM J. Appl. Math., vol. 56, pp. 1094-1106, 1996.

[26] B. L. McNaughton, C. A. Barnes, and P. Andersen, "Synaptic efficacy and EPSP summation in granule cells of rat fascia dentata studied in vitro," J. Neurophysiol., vol. 46, pp. 952-966, 1981.

[27] R. Miles and R. K. S. Wong, "Excitatory synaptic interactions between CA3 neurones in the guinea-pig hippocampus," J. Physiol., vol. 373, pp. 397-418, 1986. 
[28] R. E. Mirollo and S. H. Strogatz, "Synchronization of pulse-coupled biological oscillators," SIAM J. Appl. Math., vol. 50, pp. 1645-1662, 1990.

[29] Y. Nishura, J. Shidawara, and T. Takaishi, "Dynamics of inhibitory pulse-coupled oscillators," in Dynamical Systems and Applications. Singapore: World Scientific, 1995.

[30] C. S. Peskin, Mathematical Aspects of Heart Physiology. New York: Courant Inst. Math. Sci., 1975.

[31] R. J. Sayer, M. J. Friedlander, and S. J. Redman, "The time course and amplitude of EPSP's evoked at synapses between pairs of CA3/CA1 neurons in the hippocampal slice," J. Neurosci., vol. 10, pp. 826-836, 1990.
[32] D. Somers and N. Kopell, "Rapid synchronization through fast threshold modulation," Biol. Cybern., vol. 68, pp. 393-407, 1993.

[33] M. Tsodyks, I. Mitkov, and H. Sompolinsky, "Pattern of synchrony in inhomogeneous networks of oscillators with pulse interactions," Phys. Rev. Lett., vol. 71, pp. 1280-1283, 1993.

[34] C. V. Vreeswijk, L. F. Abbott, and G. B. Ermentrout, "When inhibition not excitation synchronizes neural firing," J. Comput. Neurosci., vol. 1, no. 313-312, 1994

Eugene M. Izhikevich, for a biography, see this issue, p. 507. 